\title{
Point spread functions for the Solar optical telescope onboard Hinode
}

\author{
S. Wedemeyer-Böhm ${ }^{1,2, \star}$ \\ ${ }^{1}$ Institute of Theoretical Astrophysics, University of Oslo, PO Box 1029 Blindern, 0315 Oslo, Norway \\ e-mail: sven.wedemeyer-bohm@astro.uio.no \\ 2 Center of Mathematics for Applications (CMA), University of Oslo, PO Box 1053 Blindern, 0316 Oslo, Norway
}

Received 20 March 2008 / Accepted 1 May 2008

\begin{abstract}
Aims. We investigate the combined point spread function (PSF) of the Broadband Filter Imager (BFI) and the Solar Optical Telescope (SOT) onboard the Hinode spacecraft.

Methods. Observations of the Mercury transit from November 2006 and the solar eclipse(s) from 2007 are used to determine the PSFs of SOT for the blue, green, and red continuum channels of the BFI. For each channel, we calculate large grids of theoretical point spread functions by convolution of the ideal diffraction-limited PSF and Voigt profiles. These PSFs are applied to artificial images of an eclipse and a Mercury transit. The comparison of the resulting artificial intensity profiles across the terminator and the corresponding observed profiles yields a quality measure for each case. The optimum PSF for each observed image is indicated by the best fit.

Results. The observed images of the Mercury transit and the eclipses exhibit a clear proportional relation between the residual intensity and the overall light level in the telescope. In addition, there is an anisotropic stray-light contribution. These two factors make it very difficult to pin down a single unique PSF that can account for all observational conditions. Nevertheless, the range of possible PSF models can be limited by using additional constraints like the pre-flight measurements of the Strehl ratio.

Conclusions. The BFI/SOT operate close to the diffraction limit and have only a rather small stray-light contribution. The $F W H M$ of the PSF is broadened by only $\sim 1 \%$ with respect to the diffraction-limited case, while the overall Strehl ratio is $\sim 0.8$. In view of the large variations - best seen in the residual intensities of eclipse images - and the dependence on the overall light level and position in the FOV, a range of PSFs should be considered instead of a single PSF per wavelength. The individual PSFs of that range allow then the determination of error margins for the quantity under investigation. Nevertheless, the stray-light contributions are found to be best matched with Voigt functions with the parameters $\sigma=0$ ".008 and $\gamma=0$ ".004, 0.'005, and 0 ".006 for the blue, green, and red continuum channels, respectively.
\end{abstract}

Key words. Sun: atmosphere - instrumentation: high angular resolution - space vehicles: instruments

\section{Introduction}

The quantitative interpretation of an observation requires a good knowledge of the properties of the utilised instrument as it can significantly affect empirically derived properties. A realistic model of the instrumental influence is of particular importance for the comparison of certain aspects of observations and numerical models, e.g., for the intensity contrast of solar granulation (Nordlund 1984).

Each optical instrument causes the radiation emitted by a point source (or an object) to be spread on the image plane of the detector. The image of an extended light source like the Sun is degraded this way and small spatial scales remain unresolved. Mathematically this phenomenon is described by means of a point spread function (PSF) or its Fourier transform, the Optical Transfer Function (OTF). Even an otherwise perfect optical telescope induces image degradation due to diffraction at the finite aperture stop. The resulting diffraction-limit effectively defines the best theoretically achievable angular resolution of a telescope.

In reality there is additional degradation due to (i) the Earth's atmosphere (for ground-based instruments) and (ii) instrumental effects. The terrestrial atmosphere introduces image degradation

^ Marie Curie Intra-European fellow of the European Commission. due to turbulence along the line of sight - the so-called seeing and scattering. Seeing has mostly an impact on the central parts of the PSF, blurring the image, but also contributes to the wings, whereas scattering becomes significant for the far wings of the PSF (Mattig 1983). It depends on the elevation of the line of sight and weather conditions (e.g., dust, aerosols or water vapour in the atmosphere).

Space-borne instruments have the advantage of not being limited by the Earth's atmosphere but still instrumental straylight has to be considered. Often the stray-light contribution is anisotropic and varies over the field of view (FOV), making it hard to derive a detailed model for the PSF. This effect is already visible in solar limb observations, where the intensity does not decrease to zero but to a finite value ${ }^{1}$. The instrumental deviations from the diffraction-limit are mostly caused by imperfections and contamination of the optical components in the telescope, e.g., dust on optical surfaces or impurity of lens material, but also microscopic scratches or micro-roughness. Also, subtle effects like the jitter of the spacecraft can contribute to the PSF. The resulting (anisotropic) stray-light increases the relative intensity contribution from the PSF wings and with it the

\footnotetext{
1 The residual intensity outside the solar disc, caused by stray-light, is often called aureole.
} 
relative height of the side-lobes, which nevertheless stay in position. With a larger contribution from the wings, the relative height of the central peak and the corresponding Strehl ratio ${ }^{2}$ decrease, while the central peak is broadened.

Constructing a realistic PSF is unfortunately a non-trivial task as often important contributions are hardly known. This is in particular true for stray-light produced inside an optical instrument (Mattig 1983). The best way to determine the properties of a telescope is, of course, direct measurement in the laboratory, but for space-borne instruments it is usually difficult to produce the same conditions as for the later operation. Another possibility of measuring the PSF is the observation of a partial solar eclipse. An illustrative example is the work by Deubner \& Mattig (1975) who used observations of a partial eclipse in 1973. They fitted the observed intensity profile across the eclipse terminator with a combination of two Gaussians (cf. Levy 1971). Nordlund (1984) argued that the wings of the spread function give rise to uncertainties, which make it problematic to retrieve a definite analytical fit of the measured profile. He showed that instead of two Gaussians, a combination of two Lorentzians also produces a fit of comparable quality. The related uncertainties can have a fundamental impact on the derived intensity contrast. Nordlund proposes a correction factor of 1.5 with respect to the results of Deubner \& Mattig. Beside these uncertainties, a fundamental problem remains as the fit of an observed terminator profile usually assumes a symmetric PSF/OTF, but can hardly account for asymmetric contributions. As we shall see in the case of SOT, a real PSF can have significant anisotropic components.

In many cases, however, suitable observations of an eclipse or a transit of Mercury or Venus (see, e.g., DeForest \& Wills-Davey, submitted) are not available. Instead, one has to estimate PSF properties from the spatial power spectral density and other ad hoc arguments. Another possibility is to estimate the PSF by comparing the intensity distribution (histograms) of a particular observation with numerical models (see., e.g., Schüssler et al. 2003; Leenaarts \& Wedemeyer-Böhm 2005; Langangen et al. 2007). Often the PSF is approximated with a linear combination of an Airy and a Lorentz function, characterised by two parameters: $\alpha$, the height of the Lorentzian relative to the height of the Airy core, and $\beta$, the $F W H M$ of the Lorentzian wings. In most cases, however, there is an extended range of possible combinations of $\alpha$ and $\beta$. More severe is the (implicit) assumption that the numerical model, which is used as reference for the fit between observed and synthetic image, is perfectly realistic.

Here a set of PSFs for the Solar Optical Telescope (SOT, Tsuneta et al. 2008; Ichimoto et al. 2008; Suematsu et al. 2008; Shimizu et al. 2007) onboard the Hinode spacecraft (Kosugi et al. 2007) is derived from observations of a Mercury transit and two eclipses for different wavelengths. The observations are described and analysed in Sect. 2. Theoretical two-dimensional PSFs are constructed by convolution of ideal diffraction-limited PSFs and Voigt functions and applied to an artificial eclipse and a Mercury transit in Sect. 3. By comparison of the resulting intensity profiles and the observational results, the best PSF is determined for each case. Discussion and conclusions follow in Sects. 5 and 6, respectively. We will systematically analyse and compare SOT observations of quiet Sun granulation to synthetic

\footnotetext{
2 The Strehl (intensity) ratio is defined as the ratio between the central peak values of a PSF and the one of an ideal, i.e., aberration-free, diffraction-limited PSF. It provides a measure for the deviation from the diffraction limit.
}

intensity images from numerical simulations in forthcoming papers.

\section{Observations}

\subsection{Filtergrams}

We obtained the observations analysed in this study with the Broadband Filter Imager (BFI) of the Solar Optical Telescope (SOT) onboard the Hinode satellite (see Sect. 1 for references). Primarily, the blue, green and red continuum channels of the BFI are considered. The central wavelengths of these filters are $450.45 \mathrm{~nm}, 555.05 \mathrm{~nm}$, and $668.40 \mathrm{~nm}$, respectively. All three filters have a bandwidth of $0.4 \mathrm{~nm}$. In addition, a few images for ,the other three BFI filters are used. These filters are CN, Ca II H, and G-band with central wavelengths of $388.35 \mathrm{~nm}, 396.85 \mathrm{~nm}$, and $430.50 \mathrm{~nm}$, respectively. The corresponding bandwidths are $0.7 \mathrm{~nm}, 0.3 \mathrm{~nm}$, and $0.8 \mathrm{~nm}$.

Observations of the Mercury transit from November 8th, 2006, and the solar eclipse of March 19, 2007, are analysed (see Fig. 1 for examples). The eclipse was partial on Earth but total for Hinode. We refer to it as total eclipse hereafter. There also occurred a partial eclipse on February 17, 2007, which was observed by Hinode, but which was not visible from the ground. This eclipse was observed with the G-band channel only but still provides useful information for this study. Additional observations of quiet Sun granulation for various positions on the disc and at the solar limb are used.

The position of the FOV and individual subregions on the solar disc is here reduced to the heliocentric position $\mu=\cos \theta$. No distinction is made between the different parts of the limb $(\mathrm{N} / \mathrm{W} / \mathrm{S} / \mathrm{E})$ as there is no noticeable difference in the limb profiles for the quiet Sun (see, e.g. Langhans \& Schmidt 2002). The pixel scale of the detector is 0 ". 05448 and thus smaller than the spatial resolution (and even the diffraction limit) of the optical system (0".2-0".3, see, e.g., Kosugi et al. 2007, and Sect. 1). As the same is still true for images with $2 \times 2$ pixel-binning, no difference in terms of contrast, etc., is to be expected. Both binned and unbinned images are thus used next to each other.

The data reduction (incl. dark subtraction and flat-fielding) was carried out with Solar Soft (Freeland et al. 2000). Dark frames and flat-fields were kindly provided by Berger. In a few cases remaining hot pixels, which often occur in compact groups of adjacent pixels, had to be removed.

Residual intensities. The intensity profiles and residual intensities in the dark areas are determined for the filtergrams from the Mercury transit, the two eclipses, and from limb observations. The residual intensity includes contributions from the PSF wings due to the optical system, but also contributions from scattered light. All results are summarised in Table 1 for different distances from the terminator (for the eclipses) and the solar limb, respectively. For the Mercury transit, the residual intensity at its disc-centre is given $\left(r \approx 5^{\prime \prime}\right)$. The table contains the values for $I_{\text {res }}$, which are the averages over all profiles, the corresponding standard variation, and (in brackets) the extrema. The value $N$ is the number of considered images. The intensity profiles are averaged parallel to the terminator/limb, resulting in a radial average for the Mercury transit.

Figure 1 illustrates the determination of the residual light level for the case of the green continuum. For the partial eclipse, however, the G-band is chosen because no other observations are available. The right column displays the intensity profiles 



Fig. 1. Hinode/SOT observations from top to bottom: a) limb observation, c) the total eclipse from March 19th, 2007, e) the partial eclipse from February 17th, 2007, and g) the Mercury transit from November 8th 2006 on the solar disc $\left(x=-52^{\prime \prime}, y=-426^{\prime \prime}, \mu=0.9\right)$ and i) at the solar limb. For all cases an exemplary image for a wavelength of $555 \mathrm{~nm}$ is selected, except for the partial eclipse which was only observed in G-band $(431 \mathrm{~nm})$. The panels to the right show intensity profiles of the image shown on the left (black solid) and for all other available images of the same kind (dark-grey). The profiles are averaged over all rows, aligned perpendicular to the terminator/limb. The intensity is normalised to the intensity of the bright solar disc close to the limb / terminator. The left half of the panels $\mathbf{h}$ ) and $\mathbf{j}$ ) show averages over the three rows and columns through the centre of the Mercury disc, whereas the radial averages can be seen in the right half. The dotted lines are for orientation only. For comparison profiles from the total eclipse observed in G-band are plotted in panel f) (dashed lines).

derived from the images on the left (black solid lines). In addition, the corresponding profiles from all other images for the same wavelength are plotted as grey lines in each panel. All profiles are averaged over pixels with the same distance to the lunar/solar/Mercury limb, respectively. There is a general trend of a higher residual intensity with increasing wavelength, although the mean G-band value is slightly higher than for the neighbouring bands (see Table 1). The variation of $I_{\mathrm{res}, \lambda}$ for images of the same wavelength is significant, clearly demonstrating that it is impossible to describe the stray-light contribution with a single 
Table 1. Residual intensities $I_{\text {res }}$ in the observations at different distances $r$ from the terminator or the solar limb, respectively. See text for details.

\begin{tabular}{|c|c|c|c|c|c|c|c|c|}
\hline $\begin{array}{l}\text { Band } \\
\lambda[\mathrm{nm}]\end{array}$ & $\begin{array}{c}\mathrm{CN} \\
388.3\end{array}$ & $\begin{array}{c}\text { Ca II H } \\
396.9\end{array}$ & $\begin{array}{l}\text { G band } \\
430.5\end{array}$ & $\begin{array}{c}\mathrm{bc} \\
450.5\end{array}$ & \multicolumn{2}{|c|}{$\begin{array}{c}\mathrm{gc} \\
555.0\end{array}$} & \multicolumn{2}{|c|}{$\begin{array}{c}\mathrm{rc} \\
668.4 \\
\end{array}$} \\
\hline \multicolumn{9}{|c|}{ Total eclipse } \\
\hline$N$ & 4 & 3 & 3 & 3 & 8 & & 3 & \\
\hline$r=5^{\prime \prime}$ & $2.0 \pm 0.2[1.7,2.3]$ & $2.3 \pm 0.6[2.0,3.0]$ & $3.4 \pm 0.9[2.8,4.4]$ & $2.9 \pm 0.4[2.4,3.2]$ & $4.7 \pm 1.5$ & {$[1.7,5.9]$} & $5.3 \pm 1.5$ & {$[3.6,6.4]$} \\
\hline$r=10^{\prime \prime}$ & $1.5 \pm 0.2[1.2,1.7]$ & $1.9 \pm 0.5[1.6,2.4]$ & $2.9 \pm 0.7[2.4,3.7]$ & $2.3 \pm 0.3[2.0,2.5]$ & $4.2 \pm 1.5$ & {$[1.1,5.3]$} & $4.7 \pm 1.5$ & {$[3.0,5.7]$} \\
\hline$r=20^{\prime \prime}$ & $0.8 \pm 0.6[0.0,1.3]$ & $1.5 \pm 0.4[1.3,2.0]$ & $2.6 \pm 0.6[2.0,3.2]$ & $1.9 \pm 0.2[1.7,2.1]$ & $3.8 \pm 1.4$ & {$[0.8,4.8]$} & $4.4 \pm 1.4$ & {$[2.8,5.2]$} \\
\hline \multicolumn{9}{|c|}{ Partial eclipse } \\
\hline$N$ & $\ldots$ & $\ldots$ & 9 & $\ldots$ & $\ldots$ & & $\ldots$ & \\
\hline$r=5^{\prime \prime}$ & $\ldots$ & $\ldots$ & $2.0 \pm 0.8[1.1,3.2]$ & $\ldots$ & $\ldots$ & & $\ldots$ & \\
\hline$r=10^{\prime \prime}$ & $\ldots$ & $\ldots$ & $1.6 \pm 0.7[0.9,2.7]$ & $\ldots$ & $\ldots$ & & $\ldots$ & \\
\hline$r=20^{\prime \prime}$ & $\ldots$ & $\ldots$ & $1.4 \pm 0.6[0.7,2.2]$ & $\ldots$ & $\ldots$ & & $\ldots$ & \\
\hline \multicolumn{9}{|c|}{ Mercury transit at solar disc-centre } \\
\hline$N$ & 8 & 5 & 5 & 5 & 5 & & 4 & \\
\hline$r=5^{\prime \prime}$ & $5.9 \pm 0.9[4.9,6.7]$ & $6.2 \pm 0.3[5.7,6.5]$ & $8.1 \pm 0.9[7.1,9.2]$ & $7.0 \pm 0.7[6.2,8.1]$ & $10.3 \pm 0.2$ & {$[10.2,10.6]$} & $11.3 \pm 0.5$ & $10.7,12.0]$ \\
\hline \multicolumn{9}{|c|}{ Mercury transit at solar limb } \\
\hline $\begin{array}{l}N \\
r=5^{\prime \prime}\end{array}$ & $\ldots$ & $\ldots$ & $\ldots$ & $\ldots$ & $\begin{array}{c}39 \\
8.0 \pm 2.2\end{array}$ & {$[6.3,13.8]$} & $\cdots$ & \\
\hline \multicolumn{9}{|c|}{ Solar limb } \\
\hline$N$ & $\ldots$ & $\ldots$ & $\ldots$ & 66 & 18 & & 20 & \\
\hline$r=5^{\prime \prime}$ & $\ldots$ & $\ldots$ & $\ldots$ & $5.3 \pm 0.7[4.3,6.8]$ & $6.9 \pm 1.1$ & {$[5.7,10.5]$} & $7.9 \pm 1.3$ & {$[5.5,9.9]$} \\
\hline$r=10^{\prime \prime}$ & $\ldots$ & $\ldots$ & $\ldots$ & $4.7 \pm 0.6[3.7,6.1]$ & $6.4 \pm 1.2$ & {$[5.1,9.9]$} & $7.2 \pm 1.2$ & {$[5.0,9.1]$} \\
\hline$r=20^{\prime \prime}$ & $\ldots$ & $\ldots$ & $\ldots$ & $4.0 \pm 0.7[2.9,5.7]$ & $6.0 \pm 1.3$ & {$[3.6,9.0]$} & $6.7 \pm 1.1$ & {$[5.0,8.3]$} \\
\hline
\end{tabular}

number (for each wavelength). The situation is much better for the Mercury transit at solar disc-centre as the individual images were taken under very similar conditions, resulting in less variation of $I_{\text {res. }}$. In the following, the individual sets of images are characterised.

Total eclipse. The mean residual intensities in the images for the total solar eclipse at a distance of 5 " from the terminator amount to $(2.9 \pm 0.4) \%$ of the mean intensity of the unocculted solar disc for the blue continuum but to $(5.3 \pm 1.5) \%$ for the red continuum, respectively. At a distance of 20 " the values reduce to $(1.9 \pm 0.2) \%$ and $(4.4 \pm 1.4) \%$. Values between $0.8 \%$ and $5.2 \%$ are found for individual (averaged) profiles.

Partial eclipse. For the partial eclipse only G-band images are available. The intensity profiles essentially form two groups, one with a residual intensity at a distance of 20 " of $0.7 \%$ to $1.0 \%$ of the solar disc intensity and the other with 1.8 to $2.2 \%$. The higher values result from images where the dark occulted area is less than $42 \%$ of the image, resulting in a higher mean count rate (see below). The low values come from images where the lunar disc covers more than half of the image and the mean count rate is low.

Mercury transit at solar disc-centre. Most images of the Mercury transit on the solar disc are taken at a heliocentric position of the Mercury disc of $\mu \approx 0.9$. The residual intensity at Mercury disc-centre is determined from the radially averaged profile across the Mercury limb with respect to the mean of surrounding solar disc area of comparable $\mu$. Close to solar disccentre $(\mu \approx 0.9)$ the following values are derived for the three continuum bands: $(7.0 \pm 0.7) \%$ (blue), $(10.3 \pm 0.2) \%$ (green), and $(11.3 \pm 0.5) \%$ (red). For the other three broadband channels the following values are found: $\mathrm{CN}(388 \mathrm{~nm}):(5.9 \pm 0.9) \%$; $\mathrm{Ca}$ $(397 \mathrm{~nm}):(6.2 \pm 0.3) \%$; and $\mathrm{Gb}(431 \mathrm{~nm}):(8.1 \pm 0.9) \%$. The Mercury transit at solar disc-centre obviously gives residual intensities that are much higher than estimated from the eclipses and from the solar limb observations. This can already be seen from the intensity profiles in Fig. 1, which decrease to much lower residual intensity values off the solar limb than compared to the centre of the Mercury disc. This is partly due to the influence of the wings of the telescope's PSF, which contribute more to the residual intensity for the Mercury disc than for an eclipse (see Sect. 4).

Mercury transit at the solar limb. In addition, there are green continuum images at the solar limb covering the beginning of the Mercury transit. The value of $I_{\text {res }, \lambda}$ refers to the mean intensity of the solar disc close to the Mercury disc to ensure a comparable $\mu$. In the left part of Fig. $1 \mathrm{j}$, profiles along the $x$ and $y$-axis through the Mercury disc-centre are shown next to the radial profiles. These also include the intensity decrease over the solar limb. At Mercury disc-centre the radial averaged profiles exhibit residual intensities of $(8.0 \pm 2.2) \%$ with extreme values of $6.3 \%$ and $13.8 \%$. Only the inner parts, which are of interest here, of the radial profiles are reliable; further away from the disc-centre, one averages the non-radial solar disc. The averages along the $x$ - and $y$-axes show very similar residual intensities (see Fig. 1j). Nevertheless, the derived values for $I_{\text {res }, \lambda}$ are smaller than for the Mercury transit at disc-centre because now a larger area of the image is dark, mapping the space beyond the solar limb. The large variation in $I_{\mathrm{res}, \lambda}$ can be explained by (i) the varying fraction of the dark area and thus the overall light level in the telescope; and (ii) the more problematic determination of a reference intensity as the Mercury disc at the solar limb spans a wider range in $\mu$.

Solar limb. The pure limb observations (i.e., without Mercury, see Fig. 1a-b) imply values for the residual intensity from $(5.3 \pm 0.7) \%$ for blue continuum to $(7.9 \pm 1.3) \%$ for the red continuum at a distance of $5^{\prime \prime}$ from the solar limb. For 20", the values decrease to $(4.0 \pm 0.7) \%$ and $(6.7 \pm 1.1) \%$, respectively. The residual intensity refers to the mean intensity of the solar disc close to the limb (average running from $-1^{\prime \prime}$ to $-2^{\prime \prime}$ ). The reference, 

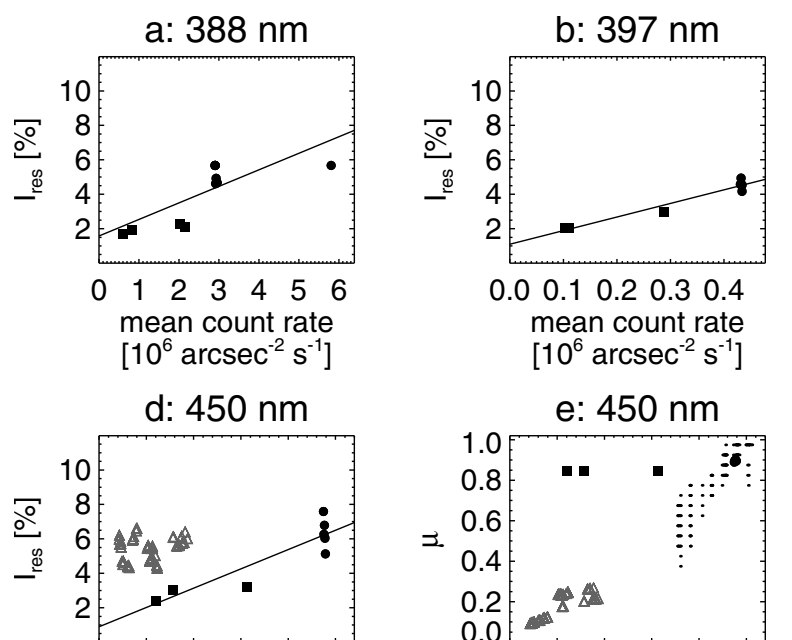

0.00 .51 .01 .52 .02 .5

mean count rate

$\left[10^{6} \operatorname{arcsec}^{-2} \mathrm{~s}^{-1}\right]$
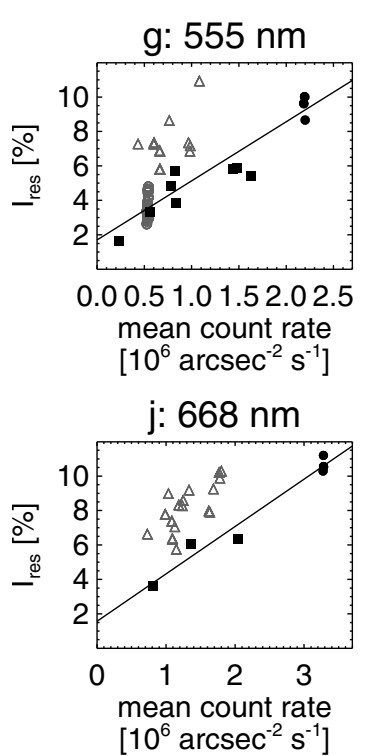
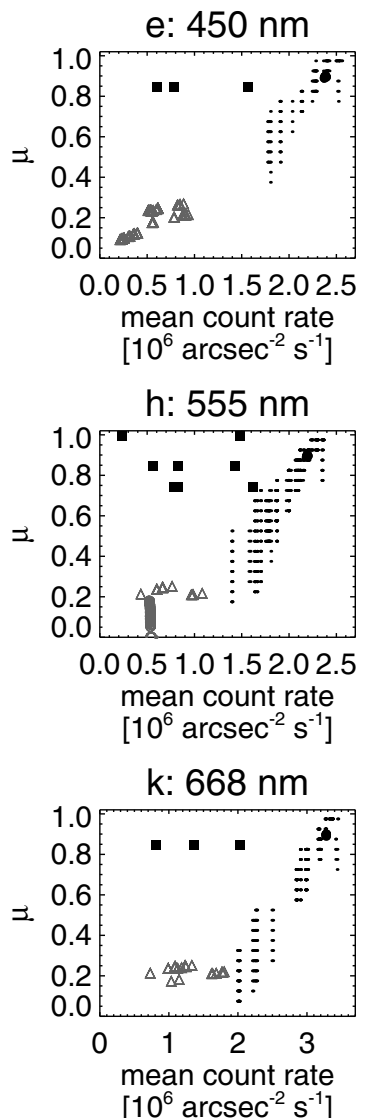
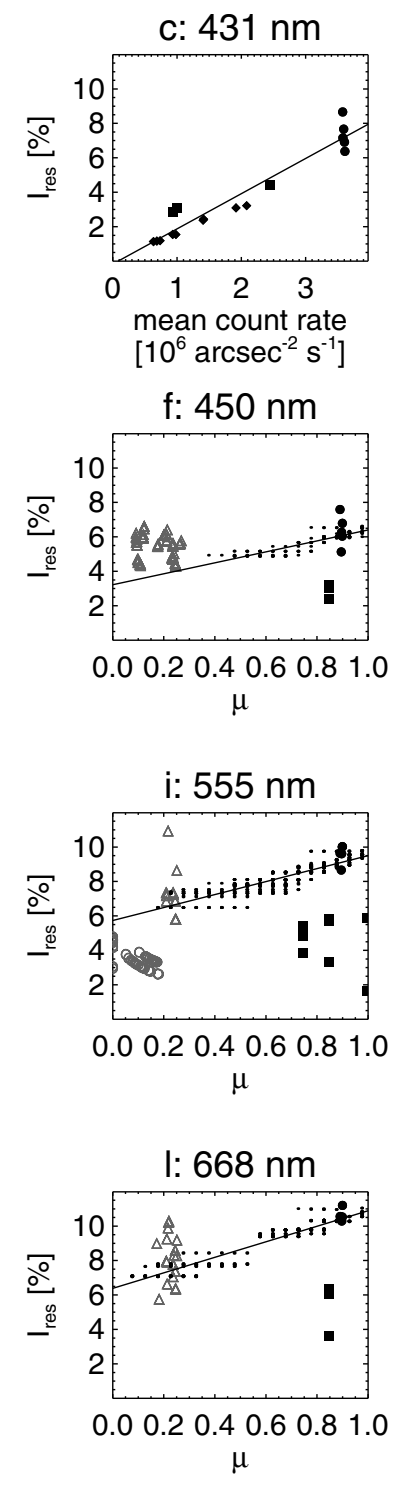

Fig. 2. Residual intensity as function of mean count rate at a distance of 5 " from the (eclipse) terminator, which corresponds to the centre of the Mercury disc. The panels in the upper row a)-c) and the leftmost column d), g), j) show the residual intensity $I_{\mathrm{res}, \lambda}$ as function of the mean count rate $C$ for the individual images of the Mercury transit (circles), the total eclipse (squares), the partial eclipse (diamonds, $431 \mathrm{~nm}$ only), and the solar limb (triangles) at all six BFI bands. Symbols are filled for $\mu>0.5$ and unfilled and dark-grey otherwise. The adopted $\mu$-value for a data point is the average over the whole individual image. An image of the solar limb thus can have $\mu<0.0$. A relation between residual intensity and mean count rate is derived by linear regression (solid lines), where the solar limb data is neglected. It is justified by the uncertainties in choosing the right reference intensity (see text for details). More information is shown for the three wavelengths $450 \mathrm{~nm}, 555 \mathrm{~nm}$, and $668 \mathrm{~nm}$. First, the heliocentric position $\mu$ is plotted as function of $C$ in the middle column $\mathbf{e}), \mathbf{h}$ ), $\mathbf{k}$ ) but not $\mathbf{b}$ ) for the eclipse and Mercury transit data and for images of "regular" quiet Sun (dots). The relation between $I_{\text {res }, \lambda}$ and $C$ is then used to estimate $I_{\text {res }, \lambda}$ from the mean count rate of the regular (unocculted) images. The results are presented in the right column $\mathbf{f}), \mathbf{i}), \mathbf{l})$. As abscissa $\mu$ is chosen. Finally, a linear regression is performed for the data points of regular granulation (solid lines). however, is problematic as the limb images often extend to $\mu \sim 0.4$ and, in some cases, even to $\mu>0.5$.

\subsection{Dependence on overall light level}

The large variation in the eclipse intensity profiles for the same wavelength is caused by the different illumination of the detector during the exposure. This "overall light level" is quantified here as the "mean count rate" $C$. It is defined as the total count number of all pixels, i.e., the full FOV of an image divided by the FOV area (in $\operatorname{arcsec}^{2}$ ) and the exposure time. For a given wavelength, an unocculted image taken at solar disc-centre has a higher value of $C$ than an image taken closer to the solar limb. The rate $C$ is reciprocally proportional to the fraction of the occulted and thus dark image area. For the images considered in this section, $C$ is highest for the Mercury transit at disc-centre. In all other cases, a significant part of the detector is dark as it images the lunar disc or the background beyond the solar limb. The same is true for the Mercury transit images at the solar limb.

The residual intensities are measured for all images at a distance of $5^{\prime \prime}$ from the terminator/solar limb. The results for the different kinds of observations are represented by different symbols in Fig. 2. For all six wavelength channels $I_{\text {res }, \lambda}$ increases linearly with $C$. We used the eclipse and Mercury transit data to derive the regression lines shown in Fig. 2. The solid lines show the result of a linear regression, which reproduce the relation quite well. The solar limb profiles are excluded here because of the uncertain reference intensity. The solar limb data is in line with the eclipse and Mercury transit when plotting the corresponding count rate instead of $I_{\text {res }}$. Obviously, a higher reference intensity is necessary to make the limb data comply with the regression lines for $I_{\text {res }}$. The dependence on $C$ indicates that the residual intensity contains contributions from anisotropic straylight, which, e.g., may originate from internal reflections in the instrument and thus clearly depend on the overall light level.

The heliocentric positions $\mu$ are plotted as function of the mean count rate for the blue, green, and red continuum in the panels e, h, and $\mathrm{k}$ of Fig. 2, respectively. The panels $\mathrm{f}, \mathrm{i}$, and 1 show the residual intensity as function of $\mu$. The Mercury transit images for the green continuum have residual intensities of $2.6 \%$ to $4.8 \%$ close to the solar limb (position of the Mercury disc at $\mu<0.2$ ), whereas $\sim 10.0 \%$ are derived close to disc-centre. It clearly demonstrates the increase of the relative residual intensity with $\mu$, related to the centre-tolimb variation of the mean intensity of solar granulation and the corresponding change of the mean count rate. 
Table 2. Estimate of the residual intensity $I_{\mathrm{res}, \lambda}$ for images of "regular" granulation in percent points. See text for details.

\begin{tabular}{cccc}
\hline \hline Wavelength & $m[\%]$ & $c[\%]$ & $\left(I_{\text {res }, \lambda}\right)_{\max }[\%]$ \\
\hline 450.45 & 3.18 & 3.22 & 6.40 \\
555.00 & 3.77 & 5.73 & 9.50 \\
668.40 & 4.51 & 6.39 & 10.90 \\
\hline
\end{tabular}
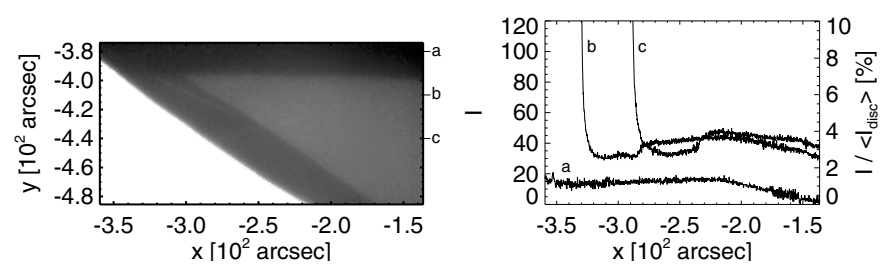

Fig. 3. Stray-light in a SOT green continuum filtergram of the total eclipse from March 19th, $2007(0.78<\mu<0.92)$ : Image (left) and profiles along the $x$-axis (right). In order to make the stray-light visible, the data range is limited to a value of 120 , which is about $10 \%$ of the mean value of the bright solar disc (lower left corner of the image). The vertical positions of the profiles are indicated by tick marks on the right side of the image. Profile a lies in a "shadow", whereas profile b crosses a "streak" at a position of $x=-300$ ".

The residual intensities in images of "regular" unocculted granulation are now estimated from the corresponding values of $C$ and the (wavelength-dependent) relation with $I_{\text {res, } \lambda}$, which was found from the Mercury transit and the eclipse data. The results for the blue, green, and red continuum are represented as dots in Fig. 2. Finally, a linear regression of the form $I_{\text {res }, \lambda}=m \cdot \mu+c$ is performed for each wavelength (solid lines in Fig. 2). The derived parameters $c$ (constant) and $m$ (slope) are listed together with the maximum value of $I_{\text {res }, \lambda}$ at $\mu=1.0$ for all three considered continuum wavelengths in Table 2 . At solar disc-centre the following residual intensities $I_{\text {res }, \lambda}$ can be expected in images of "regular" granulation: $6.4 \%, 9.5 \%$, and $10.9 \%$, for blue, green, and red continuum, respectively. The estimate is based on residual intensities at 5 " distance from the terminator of the Mercury transit and the eclipses and should thus be interpreted as upper limit only.

\subsection{Anisotropy}

A direct proof of stray-light is presented in Fig. 3. It shows a frame taken during the total eclipse, but with the displayed intensity range limited to only $10 \%$ of the mean value of the bright solar disc. There are reflections (e.g., from baffles) and a shadow at the upper edge of the detector. More "artefacts" that are not removed during the data reduction process can be found. For instance, there is a streak-like feature close to the terminator. Obviously, the stray-light is highly anisotropic. All those details are relatively subtle and might be negligible for most applications for which exact photometry is not needed. Nevertheless, the residual intensity can reach up to $4 \%$ of the solar disc intensity in this example. Similar effects are expected for the other channels, too. For instance, the red continuum channel of the BFI shows a prominent spot. Variations in the stray-light contribution due to asymmetric scattering inside the optical system have already been reported for other telescopes (e.g., Mattig 1983).

\section{Theoretical PSFs for SOT}

\subsection{Ideal diffraction-limited PSFs}

The diffraction-limited PSF of a circular homogenous illuminated aperture of diameter $d$ is described by the Airy function. For a wavelength $\lambda$ the FWHM of the central Airy disc is equal to the resolution of the telescope ${ }^{3}$

$$
\sin \alpha_{\mathrm{res}}=\frac{\lambda}{d} \text {. }
$$

For a more complicated aperture geometry the PSF can be calculated as the square of the wave number Fourier transform of the pupil shape. It has to be normalised so that the sum is equal to one. For SOT, the $50 \mathrm{~cm}$ diameter aperture, the $17.2 \mathrm{~cm}$ diameter central obscuration, and the three spiders $\left(120^{\circ}\right.$ separation, $4 \mathrm{~cm}$ wide) are taken into account. Each PSF is defined as an array with $5120^{2}$ pixels and a spatial increment of half a SOT detector pixel. The extent of the PSFs is thus wider than the minor axis of the FOV of BFI images and includes the far wings. The central lobes have the following FWHMs (cf. Eq. (1)): 0 ". 18 at $450.45 \mathrm{~nm}$ (blue); 0 ".22 at $555.05 \mathrm{~nm}$ (green); and $0 . .27$ at $668.40 \mathrm{~nm}$ (red), respectively. Suematsu (2007) kindly provided an ideal G-band PSF for the main mirror of SOT, which has a $F W H M$ of 0 ". 18 . As ideal PSFs can be scaled reciprocally with wavelength, the G-band PSF can be compared to the PSFs calculated here. They agree within the limits of numerical uncertainties.

\subsection{Non-ideal PSFS}

Here Voigt functions are used as approximation for the non-ideal PSF contributions (see Sect. 1). The final PSFs are then derived by a convolution of the ideal PSFs $\mathcal{P}_{\mathrm{dl}}$ and a Voigt function $\mathcal{V}_{\gamma}$

$\mathcal{V}_{\gamma, \sigma}(r)=(2 \pi)^{-3 / 2} \frac{\gamma}{\sigma} \int_{-\infty}^{+\infty} \frac{\mathrm{e}^{-r^{\prime 2} /\left(2 \sigma^{2}\right)}}{\left(\frac{\gamma}{2}\right)^{2}+\left(r-r^{\prime}\right)^{2}} \mathrm{~d} r^{\prime}$

which is the convolution of a Gaussian

$\mathcal{G}_{\sigma}(r)=\frac{\mathrm{e}^{-r^{2} /\left(2 \sigma^{2}\right)}}{\sqrt{2 \pi} \sigma}$

and a Lorentz function

$\mathcal{L}_{\gamma}(r)=\frac{\frac{\gamma}{2}}{\pi\left(r^{2}+\frac{\gamma^{2}}{4}\right)}$.

Here $r=\sqrt{x^{2}+y^{2}}$ is the spatial ordinate and $\gamma$ is the FWHM of the Lorentzian. The FWHM of the Gaussian is defined as $2 \sigma(2 \ln 2)^{1 / 2}$. With $u=r /(\sqrt{2} \sigma)$ and the damping parameter

$a=\frac{\gamma}{2} \frac{1}{\sqrt{2} \sigma}$

the Voigt function can also be written in the form

$\mathcal{V}(a, u)=\frac{1}{\sqrt{2 \pi} \sigma} \frac{a}{\pi} \int_{-\infty}^{+\infty} \frac{\mathrm{e}^{-u^{\prime 2}}}{a^{2}+\left(u-u^{\prime}\right)^{2}} \mathrm{~d} u^{\prime}=\frac{H(a, u)}{\sqrt{2 \pi} \sigma}$

The far wings, i.e., the PSF at large angles, are normally dominated by the Voigt function. The function is numerically reduced

\footnotetext{
${ }^{3}$ It should not be confused with the Rayleigh criterion, which is defined with an additional factor of 1.22 for the position of the first minimum surrounding the central Airy disc (approximately the first minimum of the Bessel function of the first kind).
} 

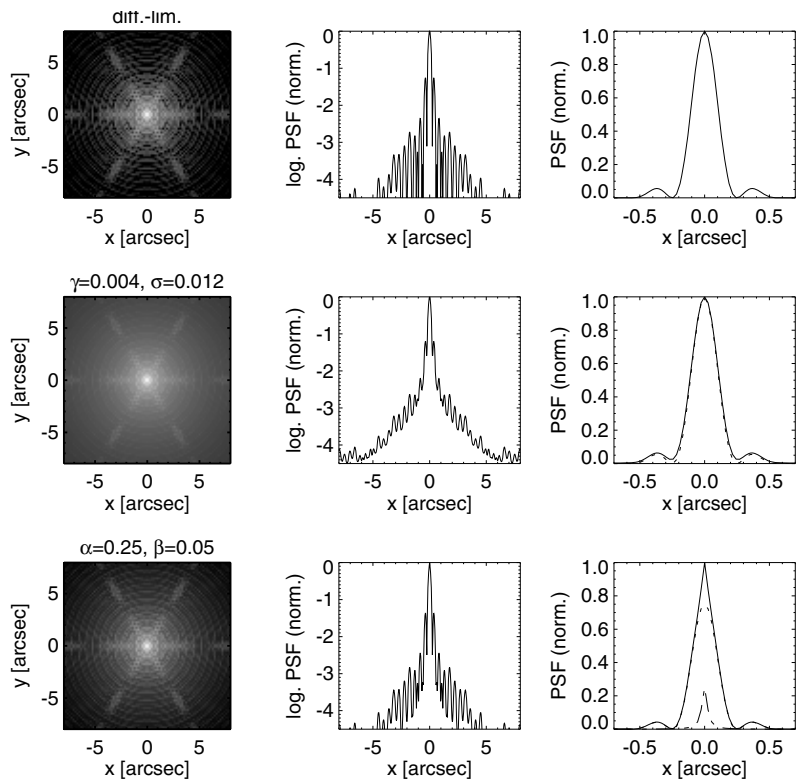

Fig. 4. Theoretical point spread functions for a wavelength of $555 \mathrm{~nm}$ : Original diffraction-limited PSF (top row), after convolution with a Voigt function with $\gamma=0$ ".004 and $\sigma=0$ ".012 (middle), and after linear combination with a Lorentzian with the parameters $\alpha=0.25$ and $\beta=0.05$ (bottom). The left columns shows the inner part of the PSFs on a logarithmic grey-scale, which covers six orders of magnitude, whereas the corresponding profiles along the $x$-axis are plotted in the middle column. The rightmost panels display the PSF core on a linear scale (solid) together with the original PSF (dotted). The dashed line in the lower right panel is the used Lorentzian profile.

to a delta peak if the $F W H M$ of the Voigt function is significantly smaller than the numerical axis increment. In that case, the convoluted PSF is identical to the original diffraction-limited one. Here PSFs with an additional Voigt function $\mathcal{V}_{\gamma, \sigma}$ are computed for a parameter grid of $\gamma$ and $\sigma$. The grid includes PSFs for which $\mathcal{P}_{\mathrm{dl}}$ is convolved with a pure Lorentzian $\mathcal{L}_{\gamma}$ for different values of $\gamma$ or with a pure Gaussian $\mathcal{G}_{\sigma}$ for different values of $\sigma$.

In some studies a linear combination of the ideal PSF and a Lorentzian of the type

$\mathcal{P}_{\mathrm{L}}(x, y)=(1-\alpha) \mathcal{P}_{\mathrm{dl}}(x, y)+\alpha \mathcal{L}_{\beta}(r(x, y))$

is used instead of a convolution. The linear approach is considered here only for comparison as it is, strictly speaking, physically not correct. Instead the convolution method is preferrable (see Sect. 5). To avoid confusion with the convolution approach the FHWM of the Lorentzian is here named $\beta$ instead of $\gamma$. A parameter grid for $[\alpha, \beta]$ is computed.

\section{Fitting observations with synthetic profiles}

Here the different theoretical PSFs for SOT (see Sect. 3) are applied to artificial idealised two-dimensional images of a Mercury transit and an eclipse. The image dimensions conform to half of the BFI detector, i.e., $2048 \times 2048$ pixels with a scale of 0 .'05448 px arcsec ${ }^{-1}$, resulting in a field of $111^{\prime \prime} 6 \times 111^{\prime \prime} .6$. The resulting synthetic degraded intensity profiles are then compared to empirical profiles derived from SOT images (see Fig. 5, for an example). The optimum PSF is the one that produces the smallest difference between observed and synthetic intensity profile.
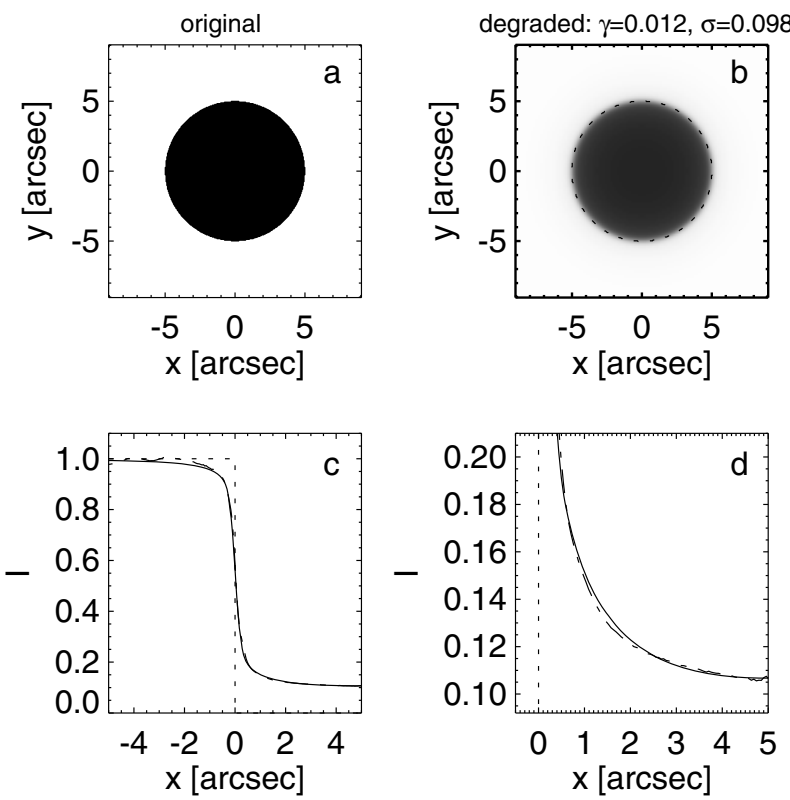

Fig. 5. Artificial observation of a Mercury transit at a wavelength of $555 \mathrm{~nm}$ : a) innermost region of the original intensity mask; b) degraded image after convolution with a non-ideal PSF that was calculated by a diffraction-limited PSF and a Voigt profile with $\gamma=0$ ". 012 and $\sigma=0$ ". 098 ; c) radial-averaged intensity profiles for the original image (dotted) and the degraded image (solid); and d) close-up of panel c). The dot-dashed line is an empirical intensity profile derived from a SOT image.

\subsection{Ideal diffraction-limited PSFs}

\subsubsection{Artificial mercury transit}

The ideal theoretical PSFs for each wavelength are applied to a simple binary mask of a bright solar background with a black Mercury disc (cf. Fig. 5). Convolution with the PSF transforms the sharp edge of the Mercury limb in the initial mask into a smooth slope. The ideal SOT PSF for a wavelength of $450.1 \mathrm{~nm}$ (blue continuum) produces a residual intensity $I_{\text {res }}$ of $1.7 \%$ (with respect to the bright solar background) at the centre of the Mercury disc in the degraded image. A simpler Airy PSF results in only $0.7 \%$ residual intensity, implying a substantial contribution due to the spiders and the central obstruction of the telescope. Already replacing the SOT PSF with its radial mean, i.e., removing the non-radial variation due to the spiders, significantly decreases the residual intensity. Quantitative results obviously demand a detailed model of the telescope properties.

Although the considered FOV is already relatively large, the residual intensity still grows asymptotically as a function of image (and PSF) size. The far wings of the PSF contribute only a little to the intensity, but nevertheless produce an asymptotic increase of $I_{\text {res }}$ (at disc-centre) by a factor of $\sim 1.1$ with respect to a field size of $111.6^{\prime \prime} \times 111.6^{\prime \prime}$. Approximately the same factor is found for $\lambda=555.0 \mathrm{~nm}$ and $668.4 \mathrm{~nm}$. The estimate is based on an analytical 2D Airy function. Under the assumption that the detailed PSFs show the same qualitative behaviour, the factor 1.1 is now multiplied to the corresponding values of $I_{\text {res }}$, resulting in residual intensities of $1.8 \%(450.1 \mathrm{~nm}), 2.2 \%(555.0 \mathrm{~nm})$, and $2.6 \%(668.4 \mathrm{~nm})$, respectively (see Table 3$)$. The proportional increase with wavelength is related to the broadening of the PSF (see the $F W H M$ ). 
Table 3. Residual intensities $I_{\text {res }}$ in the artificial diffraction-limited ${ }^{a}$ and the SOT observations ${ }^{b}$.

\begin{tabular}{|c|c|c|c|c|}
\hline$\overline{I_{\mathrm{res}}[\%]}$ & & $450 \mathrm{~nm}$ & $555 \mathrm{~nm}$ & $668 \mathrm{~nm}$ \\
\hline & Mercu & disc-centr & $\left(r=5^{\prime \prime}\right)$ & \\
\hline \multirow[t]{3}{*}{$r=5^{\prime \prime}$} & observed & $7.0 \pm 0.7$ & $10.3 \pm 0.2$ & $11.3 \pm 0.5$ \\
\hline & theoret. & 1.8 & 2.2 & 2.6 \\
\hline & difference & $5.2 \pm 0.7$ & $8.1 \pm 0.2$ & $8.7 \pm 0.5$ \\
\hline \multicolumn{5}{|c|}{ Total eclipse, different distances $r$ from lunar limb } \\
\hline \multirow[t]{3}{*}{$r=5^{\prime \prime}$} & observed & $2.9 \pm 0.4$ & $4.7 \pm 1.5$ & $5.3 \pm 1.5$ \\
\hline & theoret. & 0.6 & 0.7 & 0.8 \\
\hline & difference & $2.3 \pm 0.4$ & $4.0 \pm 1.5$ & $4.5 \pm 1.5$ \\
\hline \multirow[t]{3}{*}{$r=10^{\prime \prime}$} & observed & $2.3 \pm 0.3$ & $4.2 \pm 1.5$ & $4.7 \pm 1.5$ \\
\hline & theoret. & 0.3 & 0.3 & 0.4 \\
\hline & difference & $2.0 \pm 0.3$ & $3.9 \pm 1.5$ & $4.3 \pm 1.5$ \\
\hline \multirow[t]{3}{*}{$r=20^{\prime \prime}$} & observed & $1.9 \pm 0.2$ & $3.8 \pm 1.4$ & $4.4 \pm 1.4$ \\
\hline & theoret. & 0.2 & 0.2 & 0.3 \\
\hline & difference & $1.7 \pm 0.2$ & $3.6 \pm 1.4$ & $4.1 \pm 1.4$ \\
\hline
\end{tabular}

${ }^{a}$ All theoretical values are corrected for the influence of the far PSF wings at different distances from the terminator (see Sect. 4.1.1). ${ }^{b}$ The difference between both is due to contributions not included in the ideal PSFs, e.g., stray-light inside the telescope.

\subsubsection{Artificial eclipse}

We repeat the experiment for artificial eclipse images with a straight terminator. We consider different field sizes and also positions of the limb within the images. Again, the residual intensity, which is derived with the ideal PSF, is corrected for the asymptotic behaviour for very large bright areas due to the far wings. At a distance of 5", which corresponds to the Mercury disc radius, the following residual intensities (corrected with a factor of 1.1 for the far wings) are found to be $0.6 \%, 0.7 \%$, and $0.8 \%$ for blue, green, and red continuum, respectively (see Table 3). The values are much lower than for the Mercury experiment owing to the different geometrical situations and the different proportions of bright to occulted area. In contrast to the Mercury transit experiment, where the geometry is radialsymmetric and the occulted area is relatively small, the eclipse has a clear anisotropic geometry with a significant occulted area. At a location beyond the eclipse terminator only radiation from one side is received, whereas the centre of the Mercury disc is irradiated by the bright (solar) disc from all directions.

\subsubsection{Comparison with observations}

Comparison of the observed and artificial intensity profiles show that when using an ideal PSF (i) the average observed intensity profiles over the terminator have a less steep gradient; and (ii) the residual intensities derived from the observations are generally larger (see Table 3). This indicates that (i) the true PSFs are broader than the theoretical diffraction-limited ones; and (ii) that there is an additional contribution due to stray-light. The difference between theoretical and observed profiles decreases with distance from the terminator and thus cannot be described with just a constant intensity offset. Many profiles, averaged parallel to the terminator or just individual profiles, exhibit even a change in slope. The slope can get steeper but also reverse again in some cases (see Figs. 1 and 3). The synthetic degraded intensity profiles would fit the terminator slope of the observed (averaged) profiles better if the PSFs were broader. A broader PSF corresponds to a smaller effective aperture (or a longer wavelength, see Eq. (1)). However, even though a broader PSF would provide a better fit of the slope, the (dark) part of the profiles directly beyond the terminator is often not well reproduced. Many observed profiles do not show the theoretical asymptotic decrease toward a residual intensity, indicating that a purely diffraction-limited PSF is too simple.

\subsection{Non-ideal PSFs incl. convolution with a Voigt function}

We use PSFs calculated by convolution of the diffraction-limited PSFs and a Voigt function. Synthetic intensity profiles are again derived from degraded images of the artificial eclipse and the Mercury transit and are then compared to the observed intensity profiles. An example for the Mercury transit is displayed in Fig. 5.

A constant intensity offset $I_{0}$, which is added to the synthetic profiles $I_{\text {syn,org }}$, is introduced as a third parameter:

$I_{\text {syn }}\left(x, \gamma, \sigma, I_{\mathrm{o}}\right)=I_{\mathrm{o}}+\left(1-I_{\mathrm{o}}\right) I_{\text {syn,org }}(x, \gamma, \sigma)$.

The discrepancy between observed and synthetic profiles is measured for all parameter combinations $\left[\gamma, \sigma, I_{0}\right]$. The quality of a fit is evaluated with the quantity

$\varepsilon\left(\gamma, \sigma, I_{\mathrm{o}}\right)=\left\langle\left(\frac{I_{\mathrm{obs}}(x)-I_{\mathrm{syn}}\left(x, \gamma, \sigma, I_{\mathrm{o}}\right)}{I_{\text {syn }}\left(x, \gamma, \sigma, I_{\mathrm{o}}\right)}\right)^{2}\right\rangle_{x}$

averaged over the terminator region and the first $10^{\prime \prime}$ of the dark part in case of an eclipse and over the first $5^{\prime \prime}$ in case of the Mercury transit, respectively. This goodness-of-fit measure is in principle a sum-of-squares or least squares approach. For every $[\gamma, \sigma]$ the fitting procedure returns an error vector for the different $I_{0}$. Although the general picture remains the same when choosing a fixed $I_{\mathrm{o}}$, the minimum error is chosen for each $[\gamma, \sigma]$, effectively reducing the measured errors to $\varepsilon_{\min }(\gamma, \sigma)$. The procedure is repeated for every observed profile.

Reference case. First, a reference case is constructed to investigate the fitting routine and the quality of the selected error measure. Instead of fitting an observed intensity profile, a known synthetic profile for $\gamma=0$ ". 005 and $\sigma=0$ ". 01 is preset. The error $\varepsilon_{\min }$ forms a distinct pattern in the $\gamma-\sigma$ plane, which is shown in Fig. 6. The black track, where the error is smallest, is called here the "minimum error trench" (MET). The properties of the Voigt function make the MET bend and form a "knee". In the reference case depicted in Fig. 6, the "knee" is located at $\gamma \approx 0$ ".03 and $\sigma \approx 0$ ". 016 . Below the knee, i.e. for smaller $\sigma$, the MET follows lines of constant Strehl but also roughly lines of equal broadening of the FWHM of the PSF core with respect to the pure diffraction-limited PSF. In contrast, the part of the MET above the knee follows a line of constant damping parameter $a$ of the Voigt function (see Eq. (5)). The MET in the reference case fades again along the upper right part, indicating that the fit between the intensity profile degenerates for increasing $\sigma$. The determined best-fitting parameter pair (marked with a circle in the plot) indeed matches the preselected one, demonstrating that the method works in principle.

Fitting observed profiles. The same procedure is now used for fitting the observed intensity profiles (see Sect. 2.1). Although the MET is also clearly pronounced in Fig. 7, the best fit (not shown) is found just at the upper limit of the considered parameter range for $\sigma$. This parameter combination is clearly unreasonable as it implies a Strehl ratio of less than 0.5 , and also a FWHM of the PSF core that would be $30 \%$ broader than for the diffraction-limited PSF. It has to be attributed to imperfections of the fits due to anisotropic stray-light contributions (see 

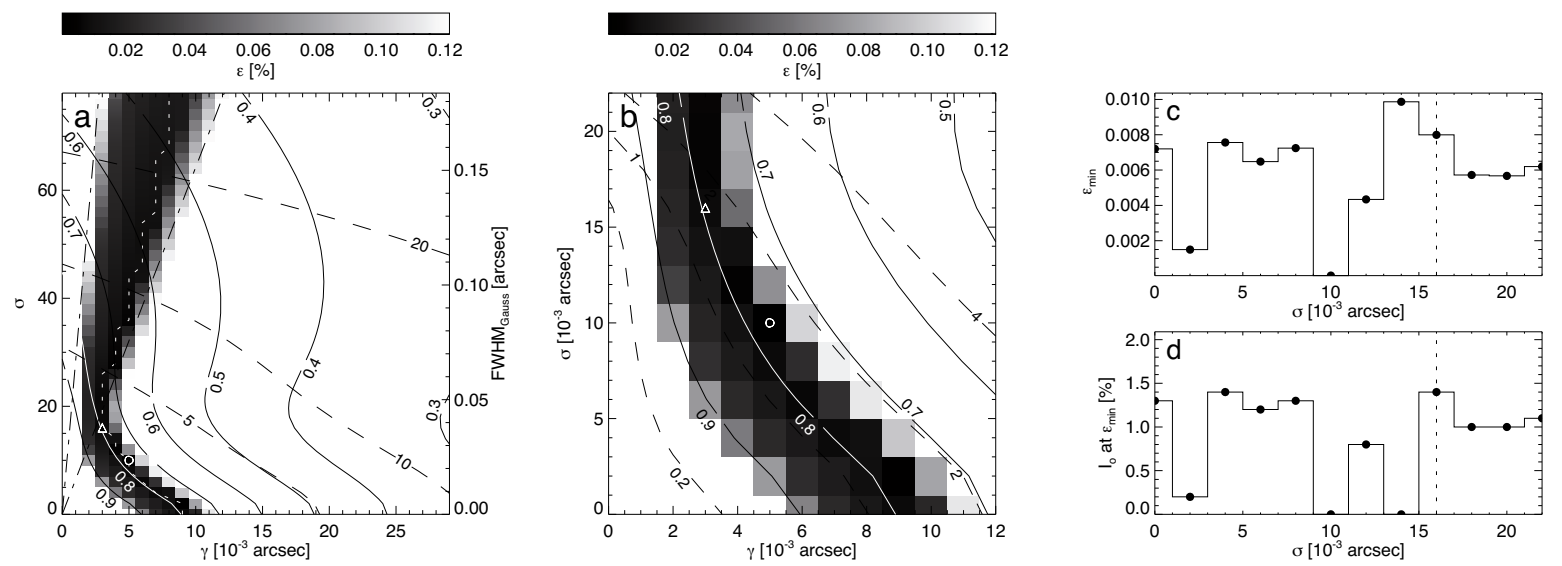

Fig. 6. Goodness $\varepsilon$ of the fits for the reference case of the Mercury transit at $\lambda=555 \mathrm{~nm}$. It represents the difference between a prescribed synthetic intensity profile for a parameter pair of $\gamma=0$ ”. 005 and $\sigma=0$ ”. 01 and all other profiles. They are derived with non-ideal PSFs calculated by convolution with a Voigt function. a) The goodness $\varepsilon$ as defined in Eq. (9) as function of $\gamma$ and $\sigma$; b) close-up of the lower left corner; c) $\varepsilon$ along the "minimum error trench" (MET, marked with white dotted line in panels a); and d) the applied intensity offset $I_{\mathrm{o}}$ along the MET. Overplotted in panels a) and b) are contours of constant Strehl ratio (solid, black and white) and of constant broadening (dashed) of the combined PSF. The broadening is given in percent points increase of the FWHM of the PSF with respect to the diffraction-limited PSF. The circles in panels a) and b) mark the parameter pair with the smallest $\varepsilon$ below the "knee" of the MET, while the "knee" is indicated by a triangle. The dot-dashed lines in panel a) are for constant Voigt damping parameter $a$, whereas the dotted vertical lines in panels c) and d) show the rough location of the MET "knee".
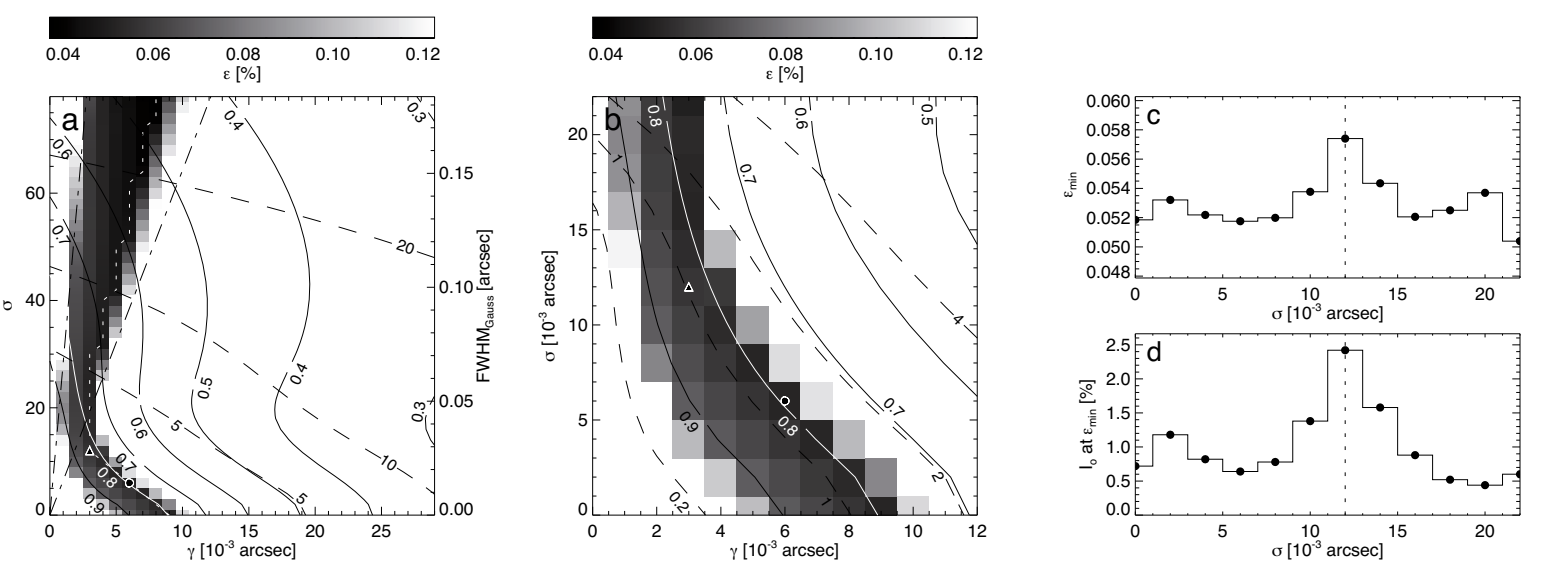

Fig. 7. Goodness $\varepsilon$ of the fits between artificial and observed intensity profiles for a selected image of the Mercury transit at $\lambda=555 \mathrm{~nm}$. The synthetic profiles are generated with non-ideal PSF that are calculated by convolution with a Voigt function. See Fig. 6 for a detailed explanation of the figure.

Sect. 5). Obviously, additional constraints are required. The spatial power spectral density of granulation images observed with SOT would, in principle, allow us to estimate the effective spatial resolution of the instrument. It would correspond to a line of constant $F W H M$ or broadening in the $\gamma$ - $\sigma$-plane (e.g., a dashed line in Fig. 7), which would restrict the relevant MET and the corresponding parameter combinations to the part below. Preliminary tests show, however, that it is difficult to determine a strict limit for the spatial resolution. Here the Strehl ratio is used as additional constraint. Based on pre-flight measurements, Tsuneta et al. (2008) and Suematsu et al. (2008) state that the individual Strehl ratios of the optical telescope assembly (OTA) and the focal-plane package (FPP) both are 0.9 or better at a wavelength of $500 \mathrm{~nm}$ and that the combined Strehl ratio is thus certainly 0.8 or better (see Tarbell et al., in prep., for details on FPP). Nevertheless, it is necessary to test the optical performance under on-orbit conditions. Indeed the MET below the "knee" follows a contour of a Strehl ratio close to 0.8 for the Mercury transit at $555 \mathrm{~nm}$ (see Fig. 7) and $668 \mathrm{~nm}$, whereas it is close to 0.85 for $450 \mathrm{~nm}$ (see linear regression section of Table 4). The METes for the eclipse are very similar and favour Strehl ratios of 0.84 to 0.86 .

In the following, the MET above the "knee" is neglected because of the excessively low Strehl ratios. Unfortunately the best parameter set $[\gamma, \sigma]$ is less obvious than in the reference case. Nevertheless, Fig. 7c points at $\gamma=0$ ".006 and $\sigma=0$ ".006 as the parameter pair that produces the best fit for that particular Mercury transit image. For the other cases, the best pair is found in different locations along the MET below the "knee". Also, the exact position of the whole MET varies slightly. Obviously, it is difficult to pin down a definite PSF that can reproduce all observations. On the other hand, the resulting parameter range represents PSFs that are very similar in terms of FWHM and Strehl ratio. The arithmetic averages and standard deviations of $\gamma$ and $\sigma$ are listed in Table 4 for all three continuum bands for the Mercury transit and the total eclipse. The variation in $\sigma$ can be quite significant, e.g., in the case of the total eclipse at $555 \mathrm{~nm}$. The variations of $\gamma$ and $\sigma$ are not independent and should rather be replaced by the deviation from the mean MET. As the MET below the "knee" follows roughly lines of constant Strehl ratio 
Table 4. Parameters ${ }^{a}$ of the convolved non-ideal PSFs that produce the best fit of the intensity profiles across the Mercury disc and the total eclipse.

\begin{tabular}{|c|c|c|c|c|c|c|}
\hline $\bar{\lambda} \lambda$ & $\overline{450.45 \mathrm{~nm}}$ & $\begin{array}{c}555.00 \mathrm{~nm} \\
\text { Mercury transit }\end{array}$ & $668.40 \mathrm{~nm}$ & $450.45 \mathrm{~nm}$ & $\begin{array}{l}555.00 \mathrm{~nm} \\
\text { Eclipse }\end{array}$ & $668.40 \mathrm{~nm}$ \\
\hline \multicolumn{7}{|c|}{ Convolution with Voigt function: best $\mathrm{fit}^{b}$} \\
\hline$\gamma_{\text {opt }}$ & $4(4 \pm 2)$ & $5(6 \pm 2)$ & $6(6 \pm 2)$ & $5(5 \pm 2)$ & $5(4 \pm 2)$ & $4(4 \pm 1)$ \\
\hline$\sigma_{\mathrm{opt}}$ & $8(8 \pm 5)$ & $8(8 \pm 4)$ & $8(8 \pm 5)$ & $7(7 \pm 5)$ & $7(7 \pm 3)$ & $7(7 \pm 3)$ \\
\hline$I_{\mathrm{o}}$ & $0.3(0.5 \pm 0.4)$ & $0.4(0.3 \pm 0.3)$ & $0.4(0.4 \pm 0.3)$ & $0.1(0.2 \pm 0.2)$ & $2.5(2.7 \pm 0.4)$ & $3.0(3.2 \pm 0.3)$ \\
\hline$b$ & $0.8(0.8 \pm 0.2)$ & $1.4(1.4 \pm 0.1)$ & $1.3(1.3 \pm 0.1)$ & $0.9(0.8 \pm 0.2)$ & $0.9(0.8 \pm 0.3)$ & $0.8(0.7 \pm 0.2)$ \\
\hline$S$ & $0.84(0.84 \pm 0.03)$ & $0.78(0.78 \pm 0.01)$ & $0.78(0.78 \pm 0.01)$ & $0.83(0.84 \pm 0.02)$ & $0.84(0.86 \pm 0.04)$ & $0.85(0.87 \pm 0.03)$ \\
\hline \multicolumn{7}{|c|}{ Linear regression of MET below the "knee" $c$} \\
\hline$\Delta \sigma / \Delta \gamma$ & $-2.672 \pm 0.218$ & $-2.228 \pm 0.164$ & $-2.277 \pm 0.148$ & $-2.587 \pm 0.309$ & $-2.528 \pm 0.237$ & $-2.619 \pm 0.097$ \\
\hline$\sigma_{C}$ & $18.2 \pm 1.6$ & $20.4 \pm 1.7$ & $21.0 \pm 1.5$ & $18.7 \pm 0.9$ & $17.7 \pm 3.2$ & $17.8 \pm 2.1$ \\
\hline$b$ & $0.8 \pm 0.2$ & $1.4 \pm 0.3$ & $1.2 \pm 0.2$ & $0.9 \pm 0.2$ & $0.9 \pm 0.3$ & $0.8 \pm 0.2$ \\
\hline$S$ & $0.85 \pm 0.03$ & $0.78 \pm 0.03$ & $0.79 \pm 0.03$ & $0.84 \pm 0.03$ & $0.86 \pm 0.04$ & $0.86 \pm 0.03$ \\
\hline \multicolumn{7}{|c|}{ Convolution with Voigt function: at MET "knee" $d$} \\
\hline$\gamma$ & $3 \pm 1$ & $4 \pm 1$ & $4 \pm 1$ & $3 \pm 1$ & $3 \pm 1$ & $3 \pm 1$ \\
\hline$\sigma$ & $13 \pm 2$ & $12 \pm 1$ & $12 \pm 1$ & $14 \pm 2$ & $12 \pm 2$ & $13 \pm 5$ \\
\hline$I_{\mathrm{O}}$ & $0.4 \pm 0.3$ & $0.4 \pm 0.6$ & $0.4 \pm 0.3$ & $0.1 \pm 0.2$ & $2.5 \pm 0.4$ & $3.0 \pm 0.4$ \\
\hline$b$ & $0.9 \pm 0.2$ & $1.5 \pm 0.2$ & $1.4 \pm 0.2$ & $1.0 \pm 0.1$ & $1.1 \pm 0.5$ & $0.9 \pm 0.2$ \\
\hline$S$ & $0.84 \pm 0.02$ & $0.78 \pm 0.01$ & $0.78 \pm 0.02$ & $0.83 \pm 0.02$ & $0.84 \pm 0.05$ & $0.85 \pm 0.04$ \\
\hline \multicolumn{7}{|c|}{ Convolution with Lorentzian $(\sigma=0)^{d}$} \\
\hline$\gamma$ & $7 \pm 1$ & $9 \pm 1$ & $9 \pm 1$ & $8 \pm 1$ & $7 \pm 1$ & $7 \pm 1$ \\
\hline$I_{\mathrm{o}}$ & $0.8 \pm 0.7$ & $0.6 \pm 0.3$ & $0.9 \pm 0.5$ & $0.1 \pm 0.2$ & $2.7 \pm 0.4$ & $3.2 \pm 0.4$ \\
\hline$b$ & $0.7 \pm 0.1$ & $1.3 \pm 0.1$ & $1.2 \pm 0.1$ & $0.8 \pm 0.1$ & $0.8 \pm 0.3$ & $0.7 \pm 0.2$ \\
\hline$S$ & $0.86 \pm 0.02$ & $0.79 \pm 0.02$ & $0.79 \pm 0.02$ & $0.84 \pm 0.02$ & $0.86 \pm 0.04$ & $0.87 \pm 0.03$ \\
\hline
\end{tabular}

${ }^{a} \gamma$ and $\sigma$ in units of $10^{-3}$ ", intensity offset $I_{\mathrm{o}}$ in $\%$, broadening factor $b$ of the $F W H M$ with respect to the ideal PSF in $\%$, and the Strehl ratio $S$. ${ }^{b}$ The values in parentheses are the arithmetic averages over the best fits for the individual observational profiles for each case. Please note that the errors in $\gamma$ and $\sigma$ are not independent (refer to the text for more details). The value in front of the parentheses is the $\varepsilon$-weighted mean value projected onto the mean MET. ${ }^{c}$ The MET below the "knee" is approximated by $\sigma_{\mathrm{MET}, \mathrm{b}}=\sigma_{C}+(\Delta \sigma / \Delta \gamma) \cdot \gamma$. The positions of the individual METs have a rms variation of $\delta \gamma \sim 0$ ".001 and $\delta \sigma \sim 0 " .002-0 " .003$, which is of the order of the grid increment. Only the METs for the eclipse at $555 \mathrm{~nm}$ show a larger variation with $\delta \gamma \sim 0$ ". 002 and $\delta \sigma \sim 0$ ".005. ${ }^{d}$ The range of reasonable PSFs is limited by the position just at the "knee" of the mean MET and on the other side by a convolution with a pure Lorentzian $(\sigma=0)$.

and broadening, the corresponding PSFs are very similar. This part of the MET can be approximated with a linear regression that accounts for all fits of the individual observations (see Table 4). The average and standard deviation of the slope $\Delta \sigma / \Delta \gamma$ and the offset $\sigma_{C}$ provide a range of METs, which can be translated into a meaningful error margin in $\gamma$ and $\sigma$. It is $\delta \gamma \sim 0$ ".001 and $\delta \sigma \sim 0$ ". $002-0$ ". 003 , which is of the order of the grid increment. Owing to the larger variation in the observational intensity profiles for the eclipse, the METs for the eclipse at $555 \mathrm{~nm}$ vary by $\delta \gamma \sim 0$ ". 002 and $\delta \sigma \sim 0$ ". 005 .

The optimum PSF for each case can now be determined from the $\varepsilon$-weighted mean value projected onto the mean MET. The resulting $[\gamma, \sigma]$ is essentially identical to the arithmetic averages or, at most, deviates in $\gamma$ by just of the order of the grid increment. For the Mercury transit a $\sigma_{\mathrm{opt}}=0$ ".008 is found for all three wavelengths, whereas $\gamma_{\mathrm{opt}}$ increases with wavelength from 0 ".004 to 0 ".006. The results for the green and red continuum are very similar in Strehl ratio $S(0.78-0.79)$ and broadening $b$ of the central PSF lobe $(1.4 \%-1.3 \%)$. In contrast the blue continuum indicates a slightly better instrument performance with $S=0.84$ and $b=0.8 \%$. The intensity offset for the optimum PSFs is derived from the average of the individual $\gamma-\sigma$-planes over all individual observations for each case at the parameter position $\left[\gamma_{\mathrm{opt}}, \sigma_{\mathrm{opt}}\right]$. It is only $0.3 \%$ to $0.4 \%$ of the mean solar disc intensity.

The eclipse case indicates an excellent instrument performance very close to the blue continuum Mercury transit case. On the other hand, larger intensity offsets of $2.5 \%$ and $3.0 \%$ are necessary for the green and blue continuum, respectively, clearly demonstrating the larger uncertainties arising from the variation among the eclipse profiles. In addition, there could be systematic differences between the Mercury transit and the eclipse observations due to the different observation dates. Some instrumental properties change with time under space conditions, causing for instance a gradual shift of the focus position (Ichimoto et al. 2008).

Finally, the range of reasonable PSFs can be defined by the limiting cases at the "knee" of the mean MET and, at the other end, by a convolution with a pure Lorentzian $(\sigma=0$, see Sect. 4.3).The indicated instrument performance is very close to those of the optimum PSFs. See Table 4 for details.

\subsection{PSFs including convolution with a Lorentzian}

For the case $\sigma=0$, the deviation from the diffraction-limit essentially reduces to a pure Lorentzian with a $F W H M$ of $\gamma$. Fitting the observed profiles requires values for $\gamma$ between 0 ".007 and 0"009 (see Table 4 and Fig. 8a). For the green and red continuum Mercury transit cases, the best fits are produced by PSFs with a Strehl ratio of $S=0.79$ and a broadening of the central lobe with respect to the diffraction-limited PSF by $1.3 \%$ and $1.2 \%$, respectively. The other cases favour Strehl ratios of 0.84 to 0.87 and a broadening of $0.7 \%$ to $0.8 \%$. The intensity offset $I_{\mathrm{o}}$ is in most cases somewhat higher than for the optimum PSFs for a convolution with a Voigt function (see Sect. 4.2) but stays of the order of $1 \%$ for the Mercury transit and up to $3.2 \pm 0.4$ for the red continuum eclipse. 

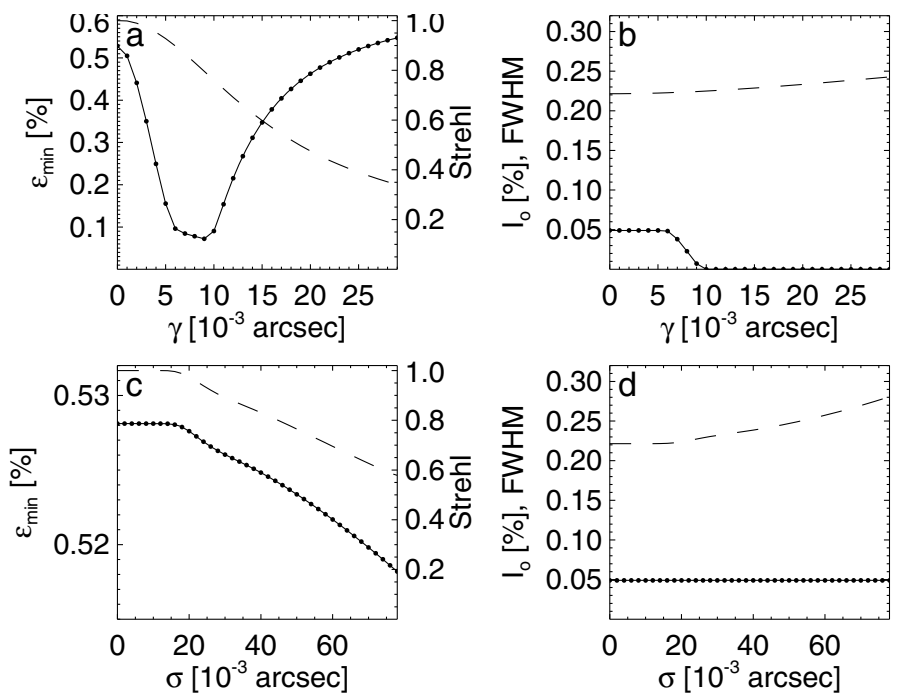

Fig. 8. Goodness $\varepsilon$ (left column, solid lines) of the fits between artificial and observed intensity profiles for the Mercury transit at $\lambda=555 \mathrm{~nm}$ and applied intensity offset $I_{\mathrm{o}}$ (right column, solid lines) for PSFs with convolution with a Lorentzian (top row) and a Gaussian (bottom row). The goodness is averaged over the individual observations. The dashed lines are the Strehl ratio in the left column and the FWHM of the combined PSF in the right column, respectively.

\subsection{PSFs including convolution with a Gaussian}

Convolution of a diffraction-limited PSF with only a pure Gaussian does produce fits to the observed intensity profiles that are usually worse than for the analogeous cases with a Lorentzian (Sect. 4.3) or better a Voigt function (Sect. 4.2). The goodness $\varepsilon$ displayed in Fig. $8 \mathrm{c}$ does not indicate any preferred parameter $\sigma$. Obviously, a pure Gaussian is generally not a suitable approximation for the non-ideal contributions to a PSF, especially for its wings. Although both the convolution with a Gaussian and Lorentzian lead to an increase in FWHM and Strehl ratio with their primary parameter ( $\sigma$ and $\gamma$, respectively), the details of the resulting PSF profiles differ. Already for small values of $\sigma$ the Gaussian causes the central lobe of the combined PSF to get so broad that the first minimum is removed and the central and secondary lobes essentially fuse. The higher order lobes are smoothed out equally fast. The Lorentzian obviously has the potential to produce profiles that are closer to reality.

\subsection{Application of non-ideal linearly combined PSFs}

Despite conceptual concerns the individual observed profiles can be fitted reasonably well when using a linear combination of an ideal PSF $\mathcal{P}_{\mathrm{dl}}$ and an additional Lorentzian (see Eq. (7) and Fig. 4). Owing to the large fluctuation among the observed intensity profiles (see Fig. 1), the best combination of $\alpha$ and $\beta$, however, varies strongly. For instance, the observed eclipse profiles at $555 \mathrm{~nm}$ are best matched with the artificial eclipse experiments when adding a Lorentzian with $\alpha=0.17 \pm 0.10$ and $\beta=0.07 \pm 0.03$, while a residuum of $\Delta I_{\text {res }}=(2.9 \pm 1.3) \%$ remains. But the range of typical parameters is extended: $\alpha=0.05-0.30$ and $\beta=0.05-0.10$ with $\Delta I_{\text {res }}=$ $(0.5-4.2) \%$. Similar values are found for the eclipse at $555 \mathrm{~nm}$ and $668 \mathrm{~nm}$, but also for the Mercury transit at $450 \mathrm{~nm}$. There are individual cases for which $\alpha$ can be up to 0.35 , but an increase in $\alpha$ is compensated by a relatively small value in $\beta$ and vice versa. In analogy to the convolution approach in Sect. 4.2, the goodness of fit shows this clear relation between $\alpha$ and $\beta$ (see Fig. 9). Obviously, there is a certain ambiguity in the parameter pair $[\alpha, \beta]$. For the other Mercury transit cases at $555 \mathrm{~nm}$ and $668 \mathrm{~nm}$ rather values around $\alpha=0.05-0.10$ and $\beta=0.15-0.25$ with $I_{\text {res }}=(0.2-1.0) \%$ prevail. Considering all profiles for all wavelengths and Mercury transit and eclipse together the following value ranges are found: $\alpha=0.15(0.05-0.30)$, $\beta=0.10(0.05-0.25), I_{\text {res }}=1.8 \%(0.0-4.5) \%$. The most frequent combination of $[\alpha, \beta]$ is $[0.25,0.05]$ with $17 \%$ of all cases, followed by $[0.20,0.05]$ and $[0.05,0.25]$ (both $13 \%$ ).

As this approach suffers from an ambiguity between $\alpha$ and $\beta$, additional constraints are necessary - just like the convolution approach in Sect. 4.2. On the other hand, some cases have to be discarded because they are unphysical, e.g., when the Strehl ratio would be greater than 1 . In general, $\beta$ should be equal to or larger than the $F W H M$ of the diffraction-limited PSF. Hence, the parameter domain left of the thick solid line in Fig. 9a, which marks $\beta=F W H M_{\mathrm{dl}}$, is invalid.

\section{Discussion}

Suitable observations. Observations of the solar limb have fundamental problems with respect to the determination of a PSF. The centre-to-limb variation induces an intensity gradient across the image, which makes a limb observation an inhomogenously illuminated and thus non-ideal test case. The corresponding stray-light level might be strongly underestimated as the overall light level is much smaller than at disc-centre (see Fig. 2). In addition, the centre-to-limb variation complicates the definition of a reference intensity for the stray-light level.

In general, it is preferable to have a homogeneous background with a well-defined proxy for the PSF. Ideal is a case which is not too different from regular observations. In this respect a Mercury transit seems to be the best available test case. The overall light level in the telescope is not significantly affected if the FOV is much larger than the occulting Mercury disc. The radial geometry is very convenient as radial averaging offers a way to deal with the anisotropy of the PSF and describes it with an average intensity offset instead. A clear drawback of course is that a Mercury transit does only provide the first 5 "of the PSF profile.

In contrast, even the far wings can be analysed for an eclipse image. On the other hand, eclipses have a clear disadvantage if there are anisotropic contributions that vary across the FOV. In that case, as found here for SOT, the exact position of an eclipse terminator in the image introduces another degree of freedom. Also, the larger relative occulted image area, which varies from image to image, make eclipse image depend much more strongly on the overall light level in the telescope.

Ideally, one should try to combine the analysis of both, Mercury transits and eclipses, whenever possible, where images away from the solar limb are preferable.

PSF models. It is not obvious which mathematical model is best for representing the non-ideal contributions of a PSF. Already Nordlund (1984) made clear that PSFs of comparable quality can be constructed from a combination of two Lorentzians instead of Gaussians. Another approach is used by DeForest \& Wills-Davey (submitted), who model the PSF of the Transition Region and Coronal Explorer (TRACE) with the sum of a narrow Gaussian core and Lorentzian wings.

An important finding is that it is indeed crucial to use realistic detailed PSFs. Ideally, one would assemble a realistic PSF by 

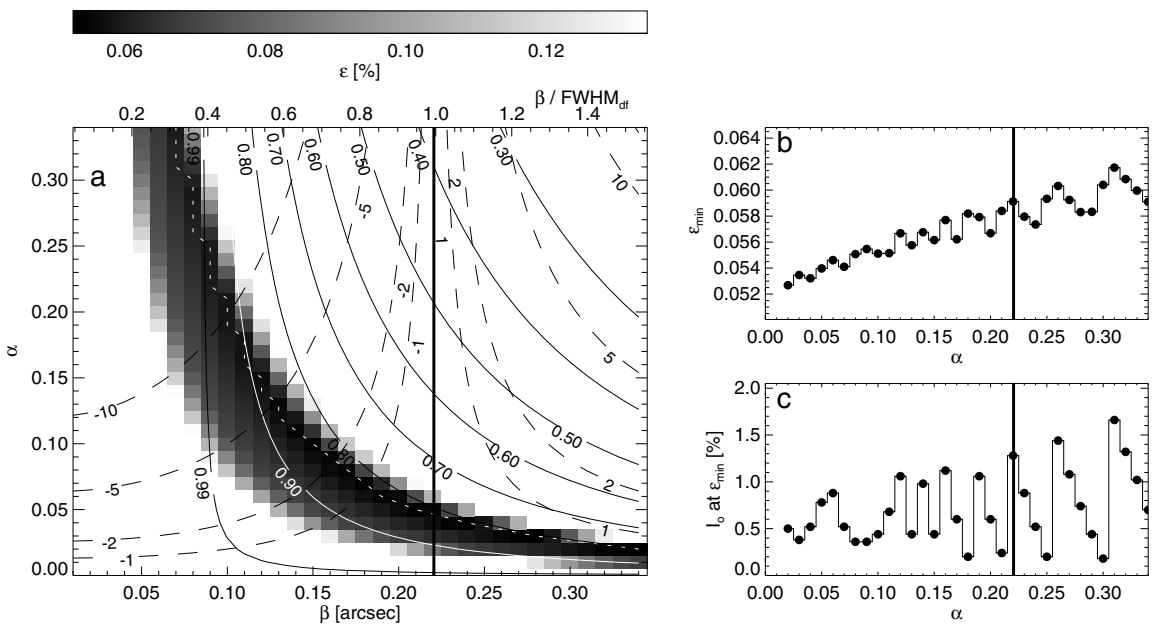

Fig. 9. Goodness $\varepsilon$ of the fits between artificial and observed intensity profiles for the Mercury transit at $\lambda=555 \mathrm{~nm}$ (cf. Fig. 6). The non-ideal PSF contribution is calculated by linear combination with a Lorentzian. a) The goodness $\varepsilon$ as defined in Eq. (9) as function of $\alpha$ and $\beta$; b) $\varepsilon$ along the "minimum error trench" (MET, marked with white dotted line in panel a); and c) the applied intensity offset $I_{\mathrm{o}}$ along the MET. Overplotted in panel a) are contours of constant Strehl ratio (solid) and of constant broadening (dashed) of the combined PSF. The broadening is given in percent points increase of the $F W H M$ of the PSF ( $F W H M_{\mathrm{PSF}}$ ) with respect to the one of the diffraction-limited PSF $\left(F W H M_{\mathrm{dl}}\right)$. All parameter combinations with a $\beta$ smaller than $F W H M_{\mathrm{dl}}$ (with except for combinations with $\alpha=0$ ) produce unphysical PSFs with $F W H M_{\mathrm{PSF}}<F W H M_{\mathrm{dl}}$. The cases are found left of the thick solid line, which marks $F W H M_{\mathrm{PSF}}=F W H M_{\mathrm{dl}}$ (in all panels). following the optical path step by step, and by adding a component for each optical element. Unfortunately, this is not possible in most cases as it requires detailed measurements and testing of each element under conditions that are very close to those of the observations. In a real optical system, chromatic aberration results in a systematic change of the optical properties with wavelength. In case of SOT, the chromatic aberration is small for the OTA (Suematsu et al. 2008) and also for the BFI and can thus usually be neglected. An exception may be simultaneous observations that span a large wavelength range, resulting in a small, but possibly significant, effect (Ichimoto et al. 2008). Even an interchange of intensity between different wavelengths is, in principle, possible. A PSF for a certain wavelength thus could gain or lose energy from other wavelengths. Here the PSFs are treated as purely monochromatic because the aforementioned effects are considered negligible in comparison to other uncertainties.

Simplifications are actually unavoidable, but one should at least account for instrumental details such as spiders and a central obstruction, as it is the case for SOT. In particular the spider legs do not only block the telescope pupil, but also scatter the incoming light at small angles, which will inevitably broaden the PSF. The strong variation in eclipse profiles and the number of potential causes, however, limit the number of meaningful free model parameters. As long as an approximation produces acceptable fits to the intensity profiles, it should thus be as simple as possible. For instance, the net effect of a possible defocus is (implicitly) included in the PSFs determined here although it cannot explicitly be separated from other effects. The introduction of a defocus term would certainly complicate the model without producing better fits of the intensity profiles.

A linear combination of a diffraction-limited PSF and a Lorentzian should only be used for sufficiently large $\beta$, which is mostly the case when the deviations are dominated by the seeing induced by the Earth's atmosphere (see, e.g., Langangen et al. 2007; Leenaarts \& Wedemeyer-Böhm 2005). For space-borne instruments, smaller values of $\beta$ and thus narrower Lorentzians can occur, as seen here, for example, of SOT. For very small $\beta$ the FWHM of the combined PSF gets smaller than the one for the diffration-limited PSF, whereas the Strehl ratio can exceed 1. In those cases, a linear combination is obviously unphysical. A Gaussian as PSF, although often used for the sake of simplicity and/or the lack of constraints on the PSF, is certainly not a good approximation, as it grossly underestimates the wing contributions. Instead, one should whenever possible prefer a convolution of a detailed ideal PSF and a Voigt function or possibly a more realistic description of the non-ideal contributions.

Limitations of the detailed PSFs for SOT. Anisotropic contributions and the complex dependence on the overall light level in the optical system and on instrumental details, such as focus position, render it simply impossible to determine only one PSF suitable for all situations. It is certainly advisable instead to use a range of detailed PSFs, which - as discussed in Sect. 4.2 for the Voigt-convolution approach - is limited by the extreme PSFs at the MET knee and for a pure Lorentzian (see Table 4). Both are still similar in terms of Strehl ratio and FWHM of the central lobe. Applying these PSFs in addition to the best-fit PSFs provide an error margin for the quantity under investigation.

The PSFs constructed here combine the instrumental effects of the optical telescope assembly (OTA) and the Broadband Filter Imager (BFI). It is not clear to what extent the results can be applied to observations with the Narrowband Filtergram Imager (NFI) and the spectro-polarimeter (SP) because the last part of the optical path inside the focal plane package differs. In principle, the PSF determination should be repeated for the other instruments, but no suitable observations of the Mercury transit were found.

A remaining uncertainty concerns the normalisation of the PSF to its integral, to which the far wings in principle contribute. The surface integrals of the Voigt functions diverge in the limit of infinite angles as the Lorentzian contribution converges too slowly. Therefore, a Lorentzian needs to be truncated to keep the total energy of the PSF finite. Here it is limited by the array size of the combined PSF. From a practical point of view, one can argue that the integrals of the PSFs in good approximation approach an asymptotic value because the growth in area is more than compensated by the steep decrease of the PSF profile. The use of a (truncated) Voigt function is still justified, considering that in view of the many sources of uncertainty it is only an approximation after all. DeForest \& Wills-Davey (submitted) truncate the non-ideal contributions to the PSF wings for TRACE by applying a Gaussian envelope. Nevertheless, a proper normalisation of the PSF requires a sufficiently large array (here a few $10^{\prime \prime}$ in each direction). As a consequence, the exact Strehl ratio 
depends on the extent of the PSF. Nevertheless, the Strehl ratios of the PSFs constructed here are of the order of 0.8 or better and thus agree with Tsuneta et al. (2008).

Stray-light estimate for SOT. Neither can one give a single number for the stray-light contribution, but at best a rough estimate for the upper limit of the stray-light contribution. As such one can use the difference between the observed residual intensity and the contribution due to the wings of the ideal (theoretical) PSF. The artificial Mercury transits for the best-fitting PSFs (see Table 4) have intensities of $6.5 \%, 10.0 \%$, and $10.8 \%$ at a distance of $5^{\prime \prime}$ from the terminator for green, blue, and red continuum, respectively. These values are close to the estimated residual intensity in "regular" granulation images (Table 2). The small Mercury disc affects the overall light level only slightly, making it a good test case. Subtracting the intensities at $5^{\prime \prime}$ derived with the diffraction-limited PSF (see Table 3 ) yields $4.7 \%$, $7.8 \%$, and $8.2 \%$, which can be considered as approximate upper limit for the stray-light contribution at that distance for high light levels. The value for the green continuum is significantly higher than judging from the eclipse image in Fig. 3, but the latter of course has a lower overall light level, which causes the residual intensities to be smaller (see Fig. 2).

Fitting procedure. Fitting a profile across a terminator is difficult as it both has an extremely steep slope and a long tail at low intensities. Depending on the chosen measure either one or the other is weighted more. As it is not obvious which approach is the best, the least squares method chosen here is kept as simple as possible. That the choice is reasonable is supported by the fact that the resulting MET in the $\gamma-\sigma$-plane indeed coincides with the pre-flight measurements of the Strehl ratio (Tsuneta et al. 2008). Removing the denominator in Eq. (9) gives more weight to the difference between observed and synthetic profile directly at the steep slope. The result is a small shift of the MET in the $\gamma-\sigma$-plane. For the Mercury transit the MET is shifted upwards by $\Delta \sigma=0$ ". $001-0{ }^{\prime \prime} .002$ and to the right by $\Delta \gamma \leq 0$ ". 001 , which is just of the order of the resolution of the $\gamma-\sigma$-plane. The Strehl ratio along the MET below the "knee" increases by just 0.01 to 0.03 . The quality measure given in Eq. (9) produces balanced fits, whereas removing the denominator can cause significant deviations in the tail. The eclipse is slightly more susceptible to changes of the quality measure $\varepsilon$, producing shifts of the MET of $\Delta \gamma=0$..001-0 ".004 and $\Delta \sigma=0$ ".002-0."008 and a change of the Strehl ratio of up to $\sim 0.10$ in some cases. Reducing the spatial range that is considered for $\varepsilon$ from $10^{\prime \prime}$ to $5^{\prime \prime}$ effects the position of the MET much less with shifts comparable to the above-mentioned uncertainties for the Mercury transit. In summary, we conclude that the Mercury transit case is less susceptible to details of the fitting procedure than the eclipse case.

Intensity offset. Although the non-ideal PSFs provide a much better fit of the observed intensity profiles than the ideal PSFs, small quantitative discrepancies remain. The remaining differences reflect the fact that the detailed PSF models constructed here are still a necessary simplification whereas the real PSFs additionally include anisotropy of the residual light and thus depend on the position in the FOV. The latter would require more constraints, which are usually - as in the present case - not available. The introduction of the constant intensity offset $I_{\mathrm{o}}$ offers a way of capturing the net effect of the position-dependence and improves the fit of the intensity profiles. Furthermore, $I_{\mathrm{o}}$ absorbs uncertainties in the determination of reference intensities and of the fitting procedure but also potential small contributions from the very far PSF wings. The values for $I_{0}$, however, are mostly only of the order of $1 \%$ of the mean solar disc intensity or less for the Mercury transit (see, e.g., Fig. 7c). The variation of the mean count rate and the anisotropy of the stray-light produce a larger remaining uncertainty, requiring intensity offsets of up to $\sim 3.6 \%$ (for the optimum PSFs with convolution of a Voigt function).

Anisotropic contributions. For an observed profile anisotropic contributions due to instrumental scattered light - although small - are expected and indeed found in case of SOT (Fig. 3). Adding an intensity contribution of the form $I_{\text {add }}(x)=x \frac{\mathrm{d}}{\mathrm{d} x} I$ to the reference case changes the slope of the synthetic intensity profiles across the terminator and removes the otherwise clear fading of the MET on the upper branch. The parameter pair producing the best fit, i.e., the minimum error, in the $\gamma-\sigma$ plane moves along the MET to the upper right to Strehl ratios that are certainly unreasonable. In reality, the slope changes are certainly more complex than the simple linear contribution tested here. Consequently, the used synthetic intensity profiles cannot perfectly match the observed profiles. There will always be a remaining (small) discrepancy. An exact match would require a detailed model of the anisotropic contributions, which would demand for more observational constraints on the PSF properties. Obviously, anisotropic contributions severly complicate the determination of a reasonable PSF.

Alternative methods. The direct determination of a (twodimensional) PSF from an observational image of an eclipse would, in principle, be superior to the forward approach presented here. In most cases, however, a direct determination is rather hopeless in view of uncertainties that arise from the PSF wings, but also from detector sensitivity, data sampling and many more. All these effects make it difficult to retrieve a definite analytical fit of a measured intensity profile. While a radialsymmetric PSF can be derived this way, but asymmetric contributions due to, e.g., instrumental stray-light are usually hard to determine. Obviously, non-radial features such as the imprint of spider legs cannot be captured this way.

One has to keep in mind that the situation is quite different from night-time observations for which stars, i.e. point sources, offer an easy way to determine a PSF. It may be possible to directly measure a two-dimensional PSF for a solar telescope by taking images of background stars far away from the solar disc, but there the conditions are fundamentally different from those of regular solar observations. As the dependence on the overall light level in the telescope effects in particular the PSF wings, a measurement outside the solar disc may produce a PSF that cannot be applied to observations of the bright solar disc. A potential technical problem is that solar telescopes and, in particular, the detectors are usually not designed for such low-intensity situations so that the low signal-to-noise ratio prevents the direct measurement of a PSF.

The analysis of very small features, in principle, provides an indicator of the effective spatial resolution and with it the FWHM of the PSF. Suematsu et al. (2008) use an intensity profile of a small G-band bright point and conclude that the OTA of SOT indeed operates close to the diffraction-limit (see also Ichimoto et al. 2008). Unfortunately, this method does not yield further information about the detailed structure of the PSF. The same is true for the spatial power spectral density of quiet Sun granulation images. 
Another common method is to determine a PSF by matching the intensity distribution based on numerical simulations of the solar atmosphere with observations. This approach should only be considered in the total absence of other independent methods as it assumes the numerical simulations to be perfectly realistic.

\section{Conclusion}

The analysis of Mercury transit and eclipse broadband filtergrams shows that SOT onboard the Hinode satellite is an excellent instrument, which performs close to the diffraction limit. In this work PSFs are constructed by a convolution of the ideal diffraction-limited PSF with a narrow Voigt function, which accounts for non-ideal contributions due to instrumental straylight. The comparison of synthetic and observed intensity profiles across the Mercury and lunar terminator unfortunately does not produce a unique solution for the best-fitting PSF, which can account for all observational conditions. Likely reasons are the anisotropy of the residual light and the dependence on the overall light level. A realistic PSF would even depend on the position on the solar disc, owing to the change of the overall light level in the telescope due to the centre-to-limb variation of the continuum intensity. Obviously, it is impossible to provide a stray-light estimate as just a single (wavelength-dependent) number.

Instead of a single PSF for each wavelength one should rather use a range of PSFs, which allow us to translate the uncertainty in PSF properties into an error margin for the quantity under investigation. Fortunately, the differences between synthetic and observed intensity profiles form a distinct relation of the Voigt parameters $\gamma$ and $\sigma$, which is named here the "minimum error trench" (MET). The empirically estimated Strehl ratio of the optical system serves as an additional constraint, limiting the range of reasonable PSFs to a part of the MET where the PSFs are very similar in terms of Strehl ratio, FWHM of the central lobe, and thus their effect on the intensity distribution. There are remaining uncertainties due to the anisotropy and light-level-dependence of the residual intensity, which result in an intensity offset during the profile fitting process. For the Mercury transit the offsets are usually only of the order of $1 \%$ of the solar disc intensity or less, whereas the eclipse images cause values of up 3.6\% with a pronounced variation among the individual images. Obviously, the Mercury transit is the more reliable test case. Nevertheless, reasonable PSF estimates can be constructed (see Table 4). They all require only narrow non-ideal contributions, indicating an excellent performance of the instrument.

Acknowledgements. The author thanks M. Carlsson, S. Tsuneta, F. Wöger, Y. Suematsu, L. Rouppe van der Voort, Ø. Langangen, O. von der Lühe, and O. Steiner for useful hints and helpful discussions. T. Berger is thanked for early flat-fields and dark frames for the SOT data reduction and also for helpful information regarding technical details of data and instrument. S. Haugan and T. Fredvik are acknowledged for their support with the Hinode data centre. An ideal G-band PSF for SOT was kindly provided by Y. Suematsu. This work was supported by the Research Council of Norway, grant 170935/V30, and a Marie Curie Intra-European Fellowship of the European Commission (6th Framework Programme, FP6-2005-Mobility-5, Proposal No. 042049). Intensive use was made of the Hinode Science Data Centre Europe hosted by the Institute of Theoretical Astrophysics of the University of Oslo, Norway. Hinode is a Japanese mission developed and launched by ISAS/JAXA, collaborating with NAOJ as a domestic partner, NASA and STFC (UK) as international partners. Scientific operation of the Hinode mission is conducted by the Hinode science team organized at ISAS/JAXA. This team mainly consists of scientists from institutes in the partner countries. Support for the post-launch operation is provided by JAXA and NAOJ (Japan), STFC (U.K.), NASA, ESA, and NSC (Norway). This research has made use of NASA's Astrophysics Data System.

\section{References}

DeForest, C. E., \& Wills-Davey, M. J., ApJ, submitted Deubner, F. L., \& Mattig, W. 1975, A\&A, 45, 167 Freeland, S., Bentley, R., \& Murdin, P. 2000, Encycl. A\&A

Ichimoto, K., Katsukawa, Y., Tarbell, T., et al. 2008, ArXiv e-prints, 804 Kosugi, T., Matsuzaki, K., Sakao, T., et al. 2007, Sol. Phys., 243, 3

Langangen, Ø., Carlsson, M., Rouppe van der Voort, L., \& Stein, R. F. 2007, ApJ, 655, 615

Langhans, K., \& Schmidt, W. 2002, A\&A, 382, 312

Leenaarts, J., \& Wedemeyer-Böhm, S. 2005, A\&A, 431, 687

Levy, M. 1971, A\&A, 14, 15

Mattig, W. 1983, Sol. Phys., 87, 187

Nordlund, A. 1984, in Small-Scale Dynamical Processes in Quiet Stellar Atmospheres, ed. S. L. Keil, 174

Schüssler, M., Shelyag, S., Berdyugina, S., Vögler, A., \& Solanki, S. K. 2003, ApJ, 597, L173

Shimizu, T., Nagata, S., Tsuneta, S., et al. 2007, Sol. Phys., 154

Suematsu, Y. 2007, priv. comm.

Suematsu, Y., Tsuneta, S., Ichimoto, K., et al. 2008, Sol. Phys., 26

Tarbell, T., et al., Sol. Phys., to be submitted

Tsuneta, S., Ichimoto, K., Katsukawa, Y., et al. 2008, Sol. Phys., 249, 167 